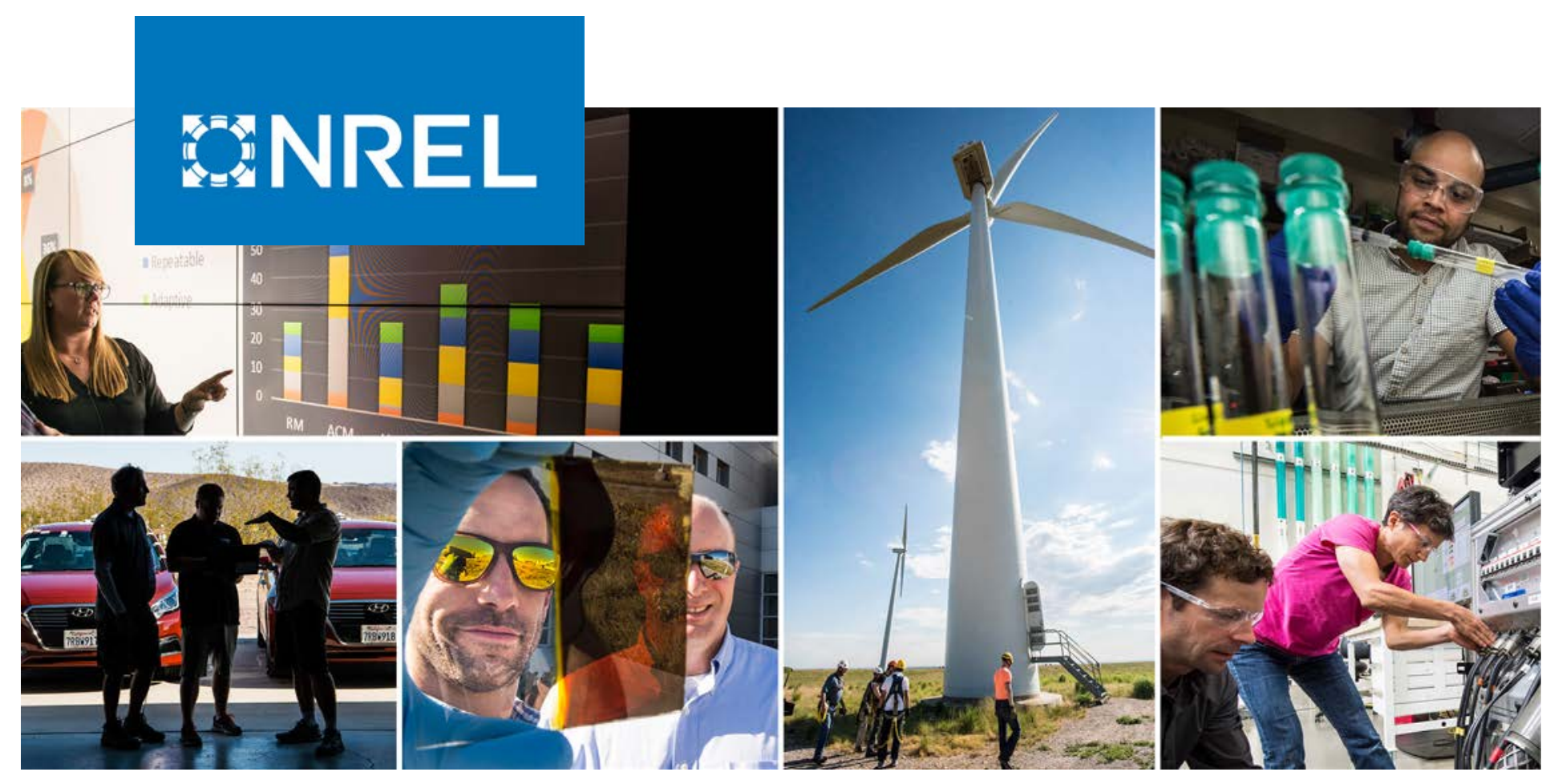

\title{
Loads Response That is Due to Wake Steering on a Pair of Utility-Scale Wind Turbines
}

Hristo Ivanov, Scott Dana, and Paula Doubrawa

National Renewable Energy Laboratory

NREL is a national laboratory of the U.S. Department of Energy

Office of Energy Efficiency \& Renewable Energy

Operated by the Alliance for Sustainable Energy, LLC

This report is available at no cost from the National Renewable Energy Laboratory (NREL) at www.nrel.gov/publications.
Technical Report

NREL/TP-5000-79187

June 2021 


\title{
FNREL
}

\section{Loads Response That is Due to Wake Steering on a Pair of Utility-Scale Wind Turbines}

\author{
Hristo Ivanov, Scott Dana, and Paula Doubrawa
}

National Renewable Energy Laboratory

\section{Suggested Citation}

Ivanov, Hristo, Scott Dana, Paula Doubrawa. 2021. Loads Response That is Due to Wake Steering on a Pair of Utility-Scale Wind Turbines. Golden, CO: National Renewable

Energy Laboratory. NREL/TP-5000-79187. https://www.nrel.gov/docs/fy21osti/79187.pdf.

NREL is a national laboratory of the U.S. Department of Energy Office of Energy Efficiency \& Renewable Energy Operated by the Alliance for Sustainable Energy, LLC

This report is available at no cost from the National Renewable Energy Laboratory (NREL) at www.nrel.gov/publications.

Contract No. DE-AC36-08GO28308
Technical Report NREL/TP-5000-79187 June 2021

National Renewable Energy Laboratory 15013 Denver West Parkway Golden, CO 80401 303-275-3000 • www.nrel.gov 


\section{NOTICE}

This work was authored by the National Renewable Energy Laboratory, operated by Alliance for Sustainable Energy, LLC, for the U.S. Department of Energy (DOE) under Contract No. DE-AC36-08GO28308. Funding provided by the U.S. Department of Energy Office of Energy Efficiency and Renewable Energy Wind Energy Technologies Office. The views expressed herein do not necessarily represent the views of the DOE or the U.S. Government.

This report is available at no cost from the National Renewable Energy Laboratory (NREL) at www.nrel.gov/publications.

U.S. Department of Energy (DOE) reports produced after 1991 and a growing number of pre-1991 documents are available free via www.OSTI.gov.

Cover Photos by Dennis Schroeder: (clockwise, left to right) NREL 51934, NREL 45897, NREL 42160, NREL 45891, NREL 48097, NREL 46526.

NREL prints on paper that contains recycled content. 


\section{Acknowledgments}

The authors of this report would like to acknowledge the wind farm owner/operator and their cooperation and assistance during this measurement campaign. In particular, the site technicians that were instrumental to completing in-situ loads calibrations. 


\section{List of Acronyms and Initialisms}

CWE

DAS

DEL

GE

GPS

HSS

IEC

LSS

$\mathrm{m}$

MW

NREL

PLC

PXI

RPM

SCADA

SLE

T1

$\mathrm{T} 2$

T3

T4

T5

TI

TN

$\mathrm{V} / \mathrm{V}$
Cold Weather Extreme

data acquisition system

damage equivalent load

General Electric

global positioning system

high-speed shaft

International Electrotechnical Commission

low-speed shaft

Wohler exponent/material slope for fatigue analysis

megawatt

National Renewable Energy Laboratory

programmable logic controller

PCI eXtensions for Instrumentation

revolutions per minute

supervisory control and data acquisition

GE designation that indicates rotor diameter of $77 \mathrm{~m}(253 \mathrm{ft})$

Turbine 1

Turbine 2

Turbine 3

Turbine 4

Turbine 5

turbulence intensity

true north

strain gage output in volt per volt 


\section{Executive Summary}

The goal of this report is to present the turbine loads resulting from a full-scale wind turbine wake steering field campaign. In the campaign's experiment, wake steering controls were applied to an upwind turbine (T2), and the downwind turbine (T3) was instrumented to measure mechanical loads.

The research turbines (T2 and T3) were GE 1.5SLE Cold Weather Extreme (CWE) turbines located on a wind farm with the prevailing wind coming from the northwest. The data collection strategy involved toggling the wake steering controls of T2 between on and off. This resulted in two databases (baseline and wake steered) with similar turbulence intensities.

Valid data were extracted and processed. The analysis involved scaling data to engineering units, applying coordinate transformations where applicable, calculating 10-minutes statistics, and calculating damage equivalent loads (DELs) to assess fatigue. Figures are shown as statistics and DELs binned by wind speed with supplemental scatter plots provided in the appendices. Results in the baseline and wake-steered databases were compared. Overall, the binned statistics showed minimal differences in loading between the two cases. However, the DELs of the wakesteered case were observed to be consistently smaller than those of the baseline for most of the turbine components.

A potential for future work was recognized based on the findings of this report. Areas of further study include an analysis for more granular loads sensitivity to different yaw offset angles and rotor wake overlap, an in-depth comparison of loads for upwind and downwind turbines, and further fatigue analysis to quantify differences observed in the experiment. 


\section{Table of Contents}

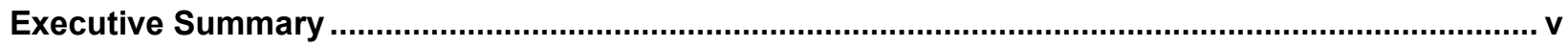

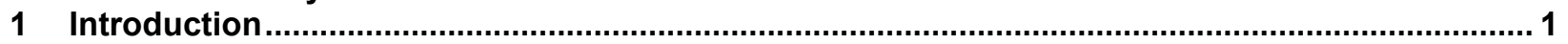

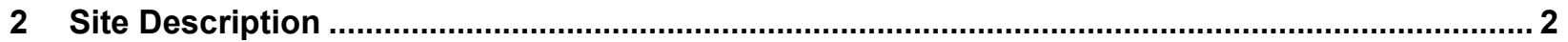

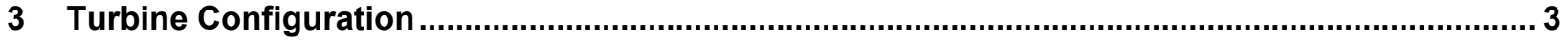

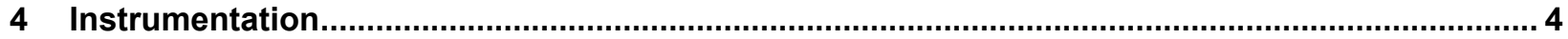

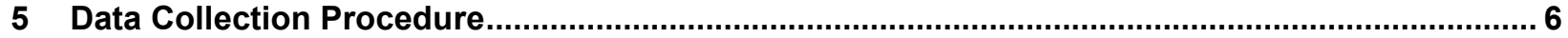

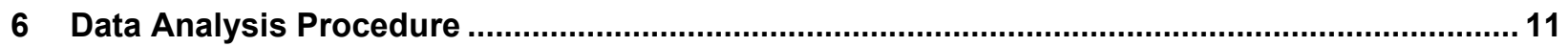

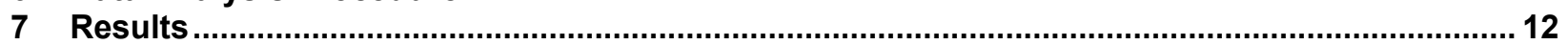

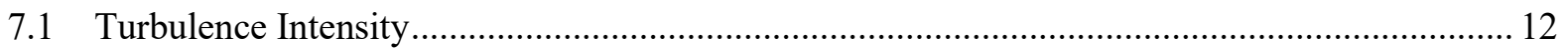

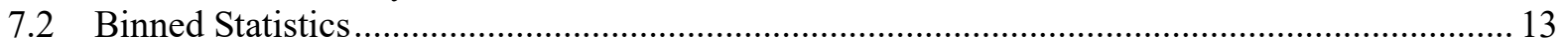

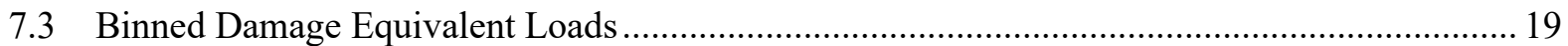

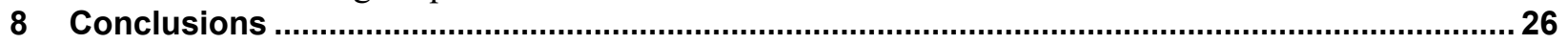

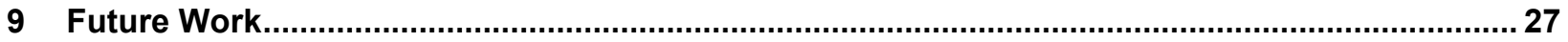

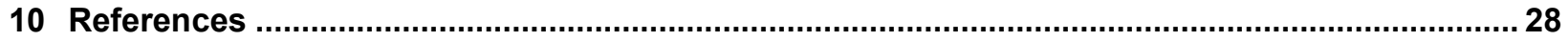




\section{List of Figures}

Figure 1. Aerial view of turbine cluster studied and meteorological instrumentation locations ....... 2

Figure 2. T2 yaw offset command schedule used by the modified controller................................... 6

Figure 3. Aerial view of T2 and T3 illustrating the measurement sector where wake steering is applied, relative to $\mathrm{T} 3$

Figure 4. Comparison of histograms of 10-minute statistics per wind speed bin for the baseline

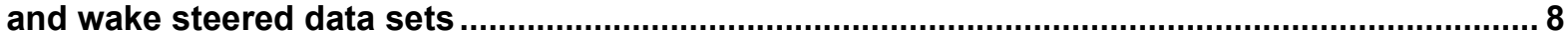

Figure 5. Capture matrix for baseline database ............................................................................... 9

Figure 6. Capture matrix for wake steered database ................................................................... 9

Figure 7. Capture matrix representing different yaw offset angles derived from wake

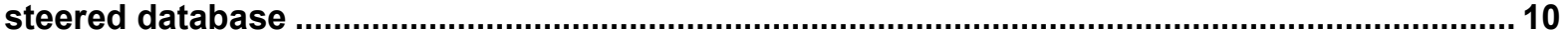

Figure 8. Comparison of turbulence intensity 10-minute statistics................................................. 12

Figure 9. Comparison of binned statistics for baseline and steered turbulence intensity ............... 13

Figure 10. Comparison of binned statistics for normalized baseline and steered blade 1 root flapwise bending moments ................................................................................................... 14

Figure 11. Comparison of normalized binned statistics for baseline and steered blade 1 edgewise

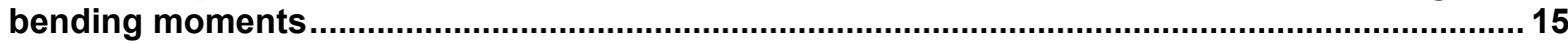

Figure 12. Comparison of normalized binned statistics for baseline and steered main shaft $M y$ bending moments................................................................................................................... 15

Figure 13. Comparison of normalized binned statistics for baseline and steered main shaft $\mathbf{M z}$

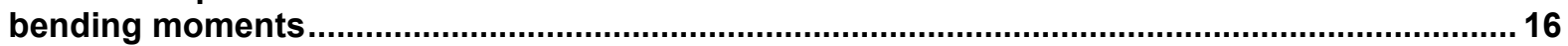

Figure 14. Comparison of normalized binned statistics for baseline and steered main shaft

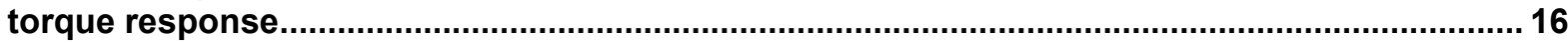

Figure 15. Comparison of normalized binned statistics for baseline and steered tower top fore-aft bending moments....................................................................................................................... 17

Figure 16. Comparison of normalized binned statistics for baseline and steered tower top side-

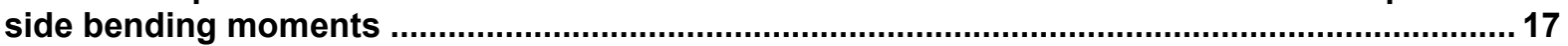

Figure 17. Comparison of normalized binned statistics for baseline and steered tower top

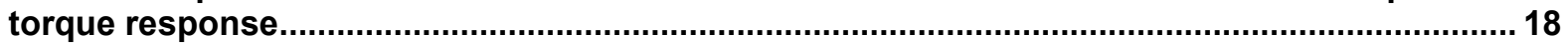

Figure 18. Comparison of normalized binned statistics for baseline and steered tower base fore-aft

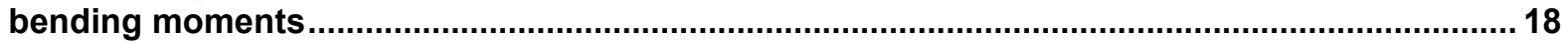

Figure 19. Comparison of normalized binned statistics for baseline and steered tower base sideside bending moments ...................................................................................................... 19

Figure 20. Comparison of normalized blade 1 root flapwise binned short-term (10-minute) damage

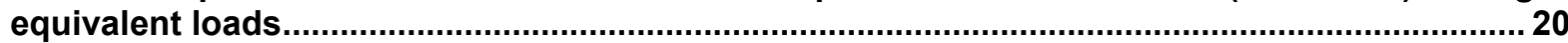

Figure 21. Comparison of normalized blade 1 root edgewise binned short-term (10-minute)

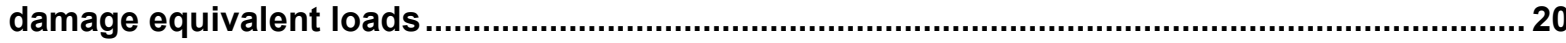

Figure 22. Comparison of normalized main shaft $M y$ binned short-term (10-minute) damage

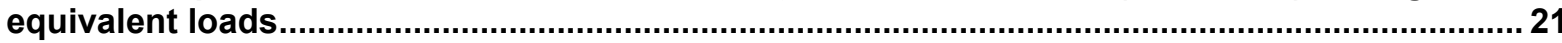

Figure 23. Comparison of normalized main shaft $\mathrm{Mz}$ binned short-term (10-minute) damage

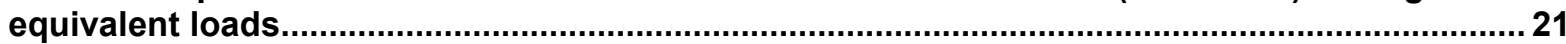

Figure 24. Comparison of normalized main shaft torque binned short-term (10-minute) damage equivalent loads.

Figure 25. Comparison of normalized tower top fore-aft binned short-term (10-minute) damage

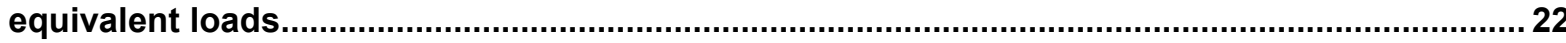

Figure 26. Comparison of normalized tower top side-side binned short-term (10-minute) damage

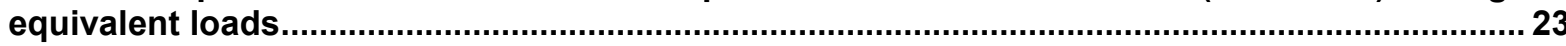

Figure 27. Comparison of normalized tower top torque binned short-term (10-minute) damage equivalent loads.

Figure 28. Comparison of normalized tower base fore-aft binned short-term (10-minute) damage equivalent loads.................................................................................................................. 24

Figure 29. Comparison of normalized tower base side-side binned short-term (10-minute) damage

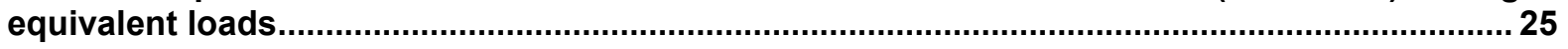

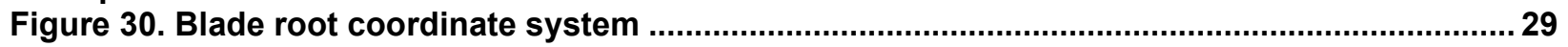

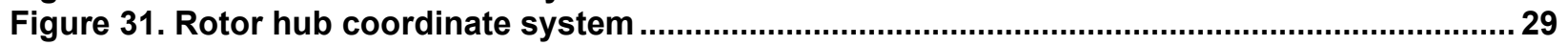




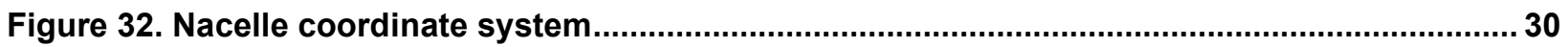

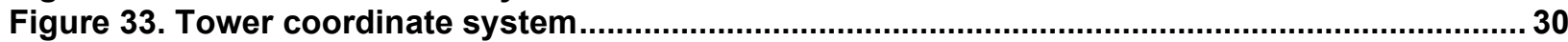

Figure 34. Comparison of 10-min statistics for baseline and steered blade 1 root flapwise bending moments normalized by absolute max of mean baseline binned statistic

Figure 35. Comparison of 10-min statistics for baseline and steered blade 1 root edgewise bending moments normalized by absolute max of mean baseline binned statistic ................... 31

Figure 36. Comparison of 10-min statistics for baseline and steered main shaft (My) bending moments normalized by absolute max of mean baseline binned statistic

Figure 37. Comparison of 10-min statistics for baseline and steered main shaft (Mz) bending moments normalized by absolute max of mean baseline binned statistic

gure 38. Comparison of 10-min statistics for baseline and steered main shaft normalized by absolute max of mean baseline binned statistic

Figure 39. Comparison of 10-min statistics for baseline and steered tower top fore-aft bending moments normalized by absolute max of mean baseline binned statistic ..

Figure 40. Comparison of 10-min statistics for baseline and steered tower top side-side bending moments normalized by absolute max of mean baseline binned statistic

Figure 41. Comparison of 10-min statistics for baseline and steered tower top torque response normalized by absolute max of mean baseline binned statistic

Figure 42. Comparison of 10-min statistics for baseline and steered tower base fore-aft bending moments normalized by absolute max of mean baseline binned statistic

Figure 43. Comparison of 10-min statistics for baseline and steered tower base side-side bending moments normalized by absolute max of mean baseline binned statistic

Figure 44. Damage equivalent load comparison of baseline and steered cases for blade 1 root flapwise bending moments normalized by the absolute max value of the baseline binned DEL

Figure 45. Damage equivalent load comparison of baseline and steered cases for blade 1 root edgewise bending moments normalized by the absolute max value of the baseline binned DEL

Figure 46. Damage equivalent load comparison of baseline and steered cases for main shaft (My) bending moments normalized by the absolute max value of the baseline binned DEL ............. 37

Figure 47. Damage equivalent load comparison of baseline and steered cases for main shaft (Mz) bending moments normalized by the absolute max value of the baseline binned DEL............. 37

Figure 48. Damage equivalent load comparison of baseline and steered cases for main shaft torque response normalized by the absolute max value of the baseline binned DEL................ 38

Figure 49. Damage equivalent load comparison of baseline and steered cases for tower top foreaft bending moments normalized by the absolute max value of the baseline binned DEL........ 38

Figure 50. Damage equivalent load comparison of baseline and steered cases for tower top sideside bending moments normalized by the absolute max value of the baseline binned DEL..... 39

Figure 51. Damage equivalent load comparison of baseline and steered cases for tower top torque response normalized by the absolute max value of the baseline binned DEL ............................ 39

Figure 52. Damage equivalent load comparison of baseline and steered cases for tower base foreaft bending moments normalized by the absolute max value of the baseline binned DEL........ 40

Figure 53. Damage equivalent load comparison of baseline and steered cases for tower base sideside bending moments normalized by the absolute max value of the baseline binned DEL..... 40 


\section{List of Tables}

Table 1. Turbine Configuration and Operational Data ...................................................................... 3

Table 2. Channel List for T3 ................................................................................................... 4

Table 3. Summary of Wind Speeds Corresponding to Baseline Absolute Maximum Mean Value of Load Variable Used for Normalization of Binned Statistics........................................................... 14

Table 4. Summary of Wind Speed Corresponding to Baseline Absolute Maximum Value of Load Variable Used for Normalization of Binned DELs .............................................................. 19 


\section{Introduction}

A cluster of five GE 1.5SLE wind turbines were used for this study (T2, T3, T4 research turbines as, and T1 and T5 as controls) to research the impact of wake steering controls applied to a wind farm [1], [2], [3]. In this field campaign, T2 and T3 were instrumented to measure turbine mechanical loads in accordance with the International Electrotechnical Commission's (IEC's) standard: Wind Turbine Generator Systems - Part 13: Measurement of Mechanical Loads (IEC 61400-13, Edition 1.0, 2015) [4], hereafter referred to as the Standard.

Based on the predominant wind direction of the site during the mechanical loads assessment period, wake steering controls were applied to T2 and the subsequent impact on the loads of T3 were studied and are presented in this report. Data from all other turbines are not presented in this report (T1, T2, T4, and T5).

All data collection and analyses were conducted following guidance from the Standard. However, this report is not meant to serve as a complete mechanical loads report as per the reporting requirements outlined in Clause 12 of the Standard. The scope of the Standard is the measurement of loads that can be used to validate a loads simulation model; the Standard is not intended to be used for the measurement of design loads or the direct comparison of data sets without a model. 


\section{Site Description}

The site's northern terrain consists of mostly flat ground. The terrain to the south is significantly sloped and considered complex terrain. The prevailing wind direction of the site is predominantly northwest, with winds coming from the southeast during the summer months. Due to this terrain the northern wind directions are favored for this study. Figure 1 provides an aerial view of the site showing the locations of T3 and neighboring turbines as well as placement of meteorological instrumentation. In accordance with the wake steering controller algorithm of T2, the National Renewable Energy Laboratory (NREL) established a measurement sector of 320 to 350 degrees relative to true north (Section 5). NREL limited assessments of loads data when the hub height wind direction averages were within the measurement sector.

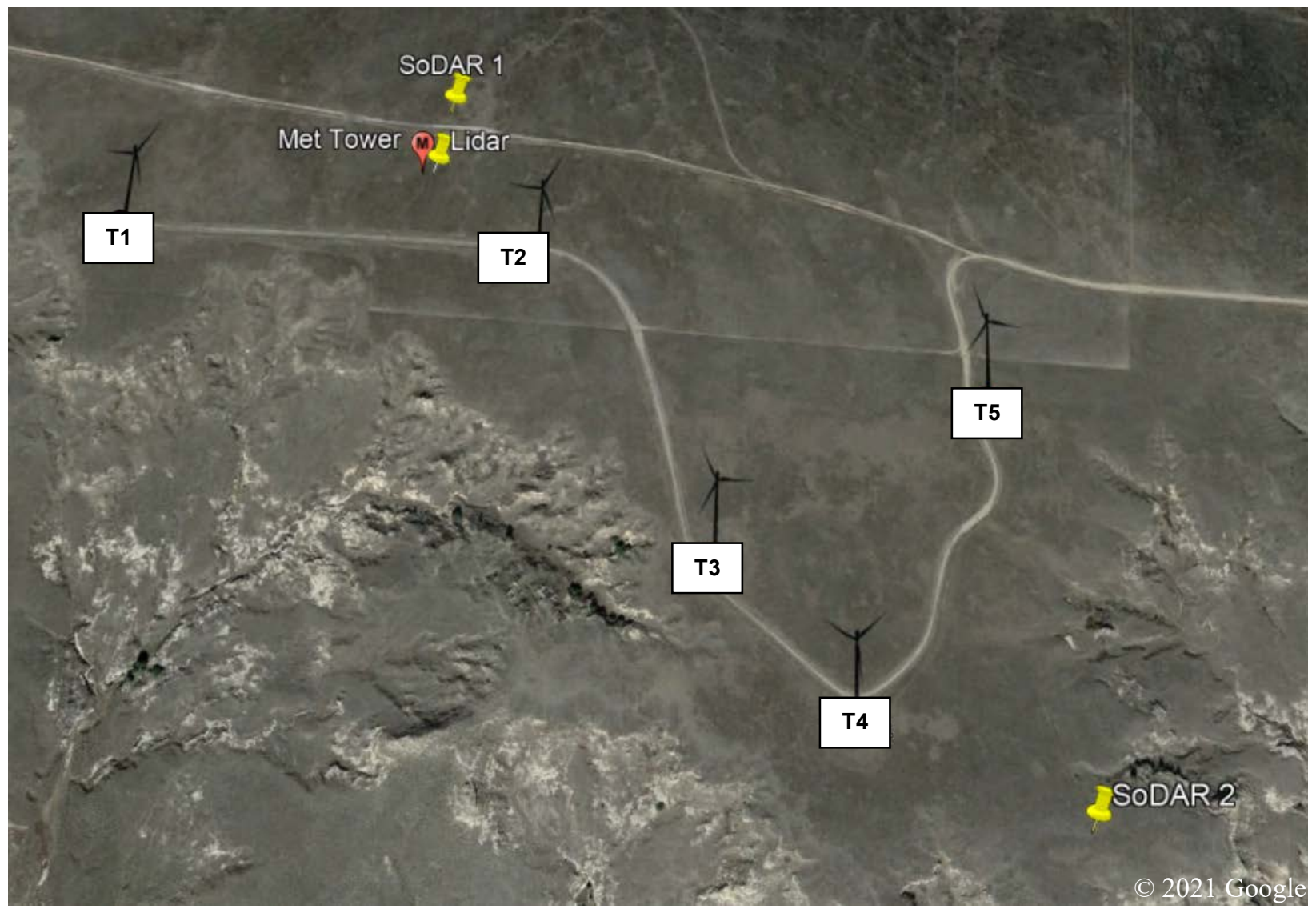

Figure 1. Aerial view of turbine cluster studied and meteorological instrumentation locations 


\section{Turbine Configuration}

The research turbines were General Electric 1.5-megawatt (MW) horizontal-axis, three-bladed, upwind machines with pitch control. Table 1 provides the key descriptive information about $\mathrm{T} 3$.

Table 1. Turbine Configuration and Operational Data

\begin{tabular}{|l|l|}
\hline Turbine Manufacturer and Address & $\begin{array}{l}\text { GE Energy } \\
300 \text { Garlington Rd., P.O. Box 648 } \\
\text { Greenville, SC 29602-0648 }\end{array}$ \\
\hline Model & GE 1.5 SLE CWE \\
\hline Rotor Diameter $(\mathrm{m})$ & 77 \\
\hline Hub Height $(\mathrm{m})$ & 80 \\
\hline Tower Type & Tubular \\
\hline Rated Electrical Power $(\mathrm{kW})$ & 1,530 \\
\hline Rated Wind Speed $(\mathrm{m} / \mathrm{s})$ & 14 \\
\hline Cut-in Wind Speed $(\mathrm{m} / \mathrm{s})$ & 3.5 \\
\hline Cut-out Wind Speed $(\mathrm{m} / \mathrm{s})$ & 25 \\
\hline Rotor Speed Range $(\mathrm{rpm})$ & $10-25$ \\
\hline Rated Rotor Speed $(\mathrm{rpm})$ & 18.3 \\
\hline Rated Generator Speed $(\mathrm{rpm})$ & 1,440 \\
\hline Gearbox Ratio & $1: 78.02$ \\
\hline Variable or Constant Speed & Variable \\
\hline Blade Pitch Angle (deg) & Variable \\
\hline Number of Blades & 3 \\
\hline Blade Make and Type & Tecsis1760 GE 37C \\
\hline Control System & Bachmann PLC \\
\hline Type Class & II-S $(52.5 \mathrm{~m} / \mathrm{s}, 50-y e a r$ gust $)$ \\
\hline
\end{tabular}




\section{Instrumentation}

The data acquisition system (DAS) used was a National Instruments PXI real-time scan engine with a distributed EtherCAT chassis and $\mathrm{C}$-series signal conditioning modules. This system uses a deterministic EtherCAT protocol that allows for exact synchronization of all signals. Every sample was GPS timestamped. The DAS hardware was paired with a PC running customdeveloped and validated LabVIEW code that was used to apply scale factors, control sampling rates, and write data files to a local hard drive.

Data were collected in accordance with the Standard. Data were sampled at $1 \mathrm{kHz}$ (the scan rate of the DAS) and then down-sampled to $50 \mathrm{~Hz}$ for storage and post-processing. Data files have a 10-minute time window. The data collection process was repeated for consecutive time windows. In addition, standard deviation, minimum, and maximum statistics for each averaging period were determined and stored.

Table 2 provides the instrumentation list with channel names and quantities measured.

Table 2. Channel List for T3

\begin{tabular}{|l|l|l|}
\hline Channel Name & Instrument & Quantity \\
\hline T3_TB_Bending_1 & Strain gage & Strain, VN \\
\hline T3_TB_Bending_2 & Strain gage & Strain, V/V \\
\hline T3_Active_Power & Watt meter & Electrical power, kW \\
\hline T3_Reactive_Power & Watt meter & Reactive power, kW \\
\hline T3_Power_Factor & Watt meter & Ratio, (-) \\
\hline T3_Pitch_Angle & Turbine controller output & Position, deg \\
\hline T3_Nacelle_WD & Turbine controller output & Position, deg TN \\
\hline T3_Rotor_Speed & Turbine controller output & LSS velocity, RPM \\
\hline T3_Power & Turbine controller output & Electrical power, kW \\
\hline T3_Torque & Turbine controller output & LSS torque, kN-m \\
\hline T3_Turbine_Status & Turbine controller output & Status, (-) \\
\hline T3_Wind_Speed & Turbine controller output & Velocity, m/s \\
\hline T3_TT_Bending_1 & Strain gage & Strain, V/V \\
\hline T3_TT_Bending_2 & Strain gage & Strain, V/V \\
\hline T3_TT_Torque & Strain gage & Strain, V/V \\
\hline T3_MS_Bending_1 & Strain gage & Strain, VNV \\
\hline T3_MS_Bending_2 & Strain gage & Strain, VNV \\
\hline T3_MS_Torque & Strain gage & Strain, V/V \\
\hline T3_Rotor_Azimuth & Absolute encoder & Rotor azimuthal position, deg \\
\hline T3_HSS_RPM & Proximity sensor & HSS velocity, RPM \\
\hline T3_B1_Flap & Strain gage & Strain, V/V \\
\hline & & \\
\hline
\end{tabular}




\begin{tabular}{|l|l|l|}
\hline Channel Name & Instrument & Quantity \\
\hline T3_B1_Edge & Strain gage & Strain, V/V \\
\hline T3_B2_Flap & Strain gage & Strain, V/V \\
\hline T3_B2_Edge & Strain gage & Strain, V/V \\
\hline T3_Nacelle_Yaw_Position & Absolute encoder & Position, deg TN \\
\hline T3_Blade1_Pitch_Indep & Absolute encoder & Position, deg TN \\
\hline
\end{tabular}

Inflow conditions were measured using several different instruments during the field campaign. For this analysis, inflow measurements were taken from the nacelle-mounted wind vane and cup anemometer. The T3 nacelle-mounted cup anemometer was used, as no other instrumentation could provide the wind speed downwind of T2. Specifics on wind direction filtering are given in Section 5.

Tower base gages were located above the tower base's transition platform. Tower top gages were located above the yaw deck platform at the top of the tower. Tower base and tower top bending scale factors were determined using the rotor overhang moment. Tower top torque coefficient was determined analytically using tower dimensions and material properties.

Yaw position was measured on the yaw gear using an absolute encoder with a companion gear that matched the yaw drive gear profile. Appropriate scale factors were applied to output yaw position relative to true north.

The main shaft gage signals were located approximately 1,000-mm downwind of the main bearing centerline, and they were connected to a wireless signal transmission system used to broadcast the signals to a receiver that provided analog inputs to the DAS. Main shaft bending moment scale factors were determined analytically using the main shaft dimensions, material properties, gage factor, and bridge completion type. Main shaft torque was determined in-situ using independently measured turbine power and rotor speed.

Azimuth was measured using an absolute encoder located on the gearbox slipring. Zero azimuth was aligned with Blade 1 pointing down at the six o'clock position.

Blade signals were measured at the blade root, 1,500-mm from the face of the pitch ring, with full bending bridges in orthogonal orientations. Blade flat and edge bending moment coefficients were determined using the blade overhang moment through a slow rotor roll procedure at two different pitch angles. 


\section{Data Collection Procedure}

Data collection was facilitated using the instrumentation described in the previous section. In accordance with the Standard, valid data are defined as coming from 10-minute periods of time when all loads signals are working properly, the turbine is operating normally, and it is producing power for the full 10-minute period. Using these protocols, the data collection period was defined as December 10, 2019, through February 16, 2020.

In this field campaign, the experiment was conducted by applying wake steering controls to the upwind turbine (T2). Based on the experience gained from previous wake steering studies ([1], [2]), the wind vane input signal of T2 was modified to achieve the desired yaw offset commands as presented in Figure 2. This figure illustrates the commanded yaw offset according to wind direction and wind speed (U) measured on T2. For wind speeds below $9 \mathrm{~m} / \mathrm{s}$ the controller attempts to follow the commanded yaw offset schedule indicated by the red line in the figure. The measurement sector where wake steering was applied in relation to T2 and T3 is shown in Figure 3. A detailed explanation of wake steering control logic is outside of the scope of this report, but additional information, related to this experiment, can be found in [2] and [3].

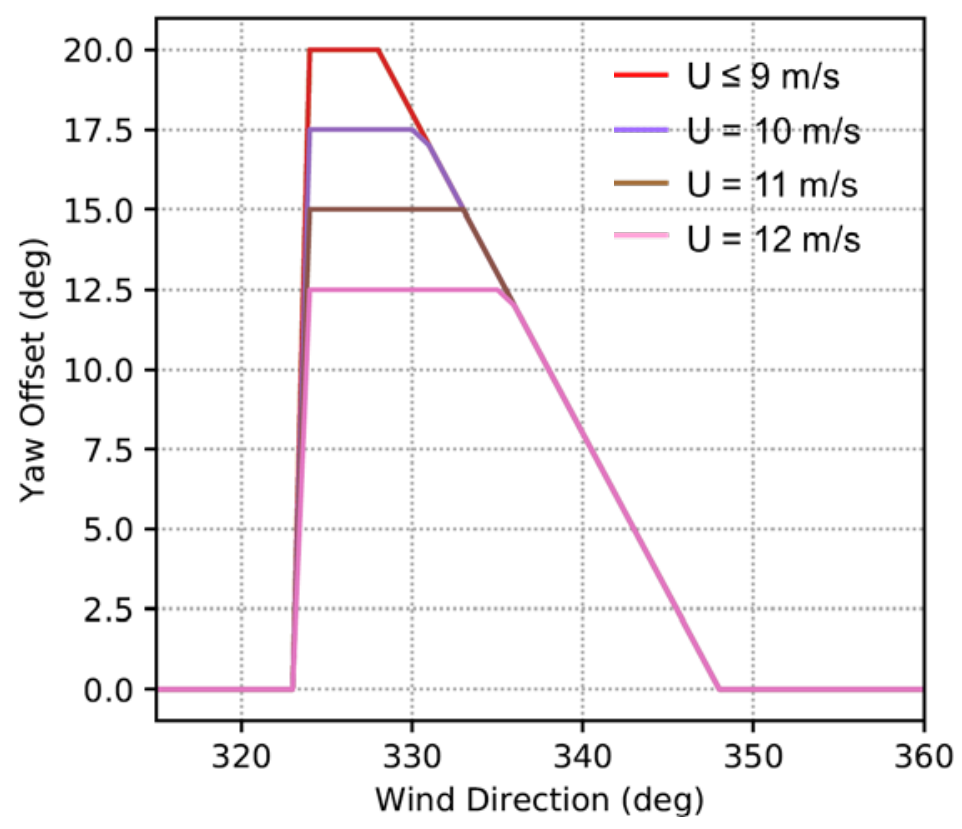

Figure 2. T2 yaw offset command schedule used by the modified controller 


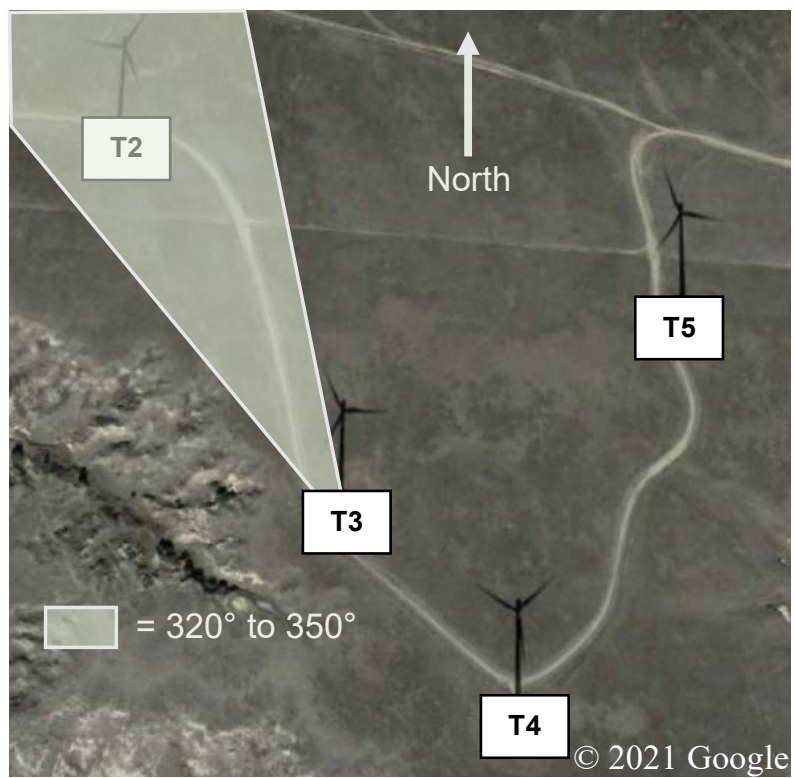

Figure 3. Aerial view of T2 and T3 illustrating the measurement sector where wake steering is applied, relative to $\mathrm{T} 3$

To create a baseline case, the modified wind vane controller alternated hourly between states of wake steering control enabled and disabled, thus creating periods when wake steering was not applied. This resulted in the creation of two databases:

1. Baseline - periods with conventional turbine controls when wake steering was not applied.

2. Steered - periods when the modified controller was sending yaw offset commands to apply wake steering.

A comparison of the wind speed bin counts $(1 \mathrm{~m} / \mathrm{s}$ bin widths, 10 -minute averages $)$ for the valid data in this analysis are provided in Figure 4. 


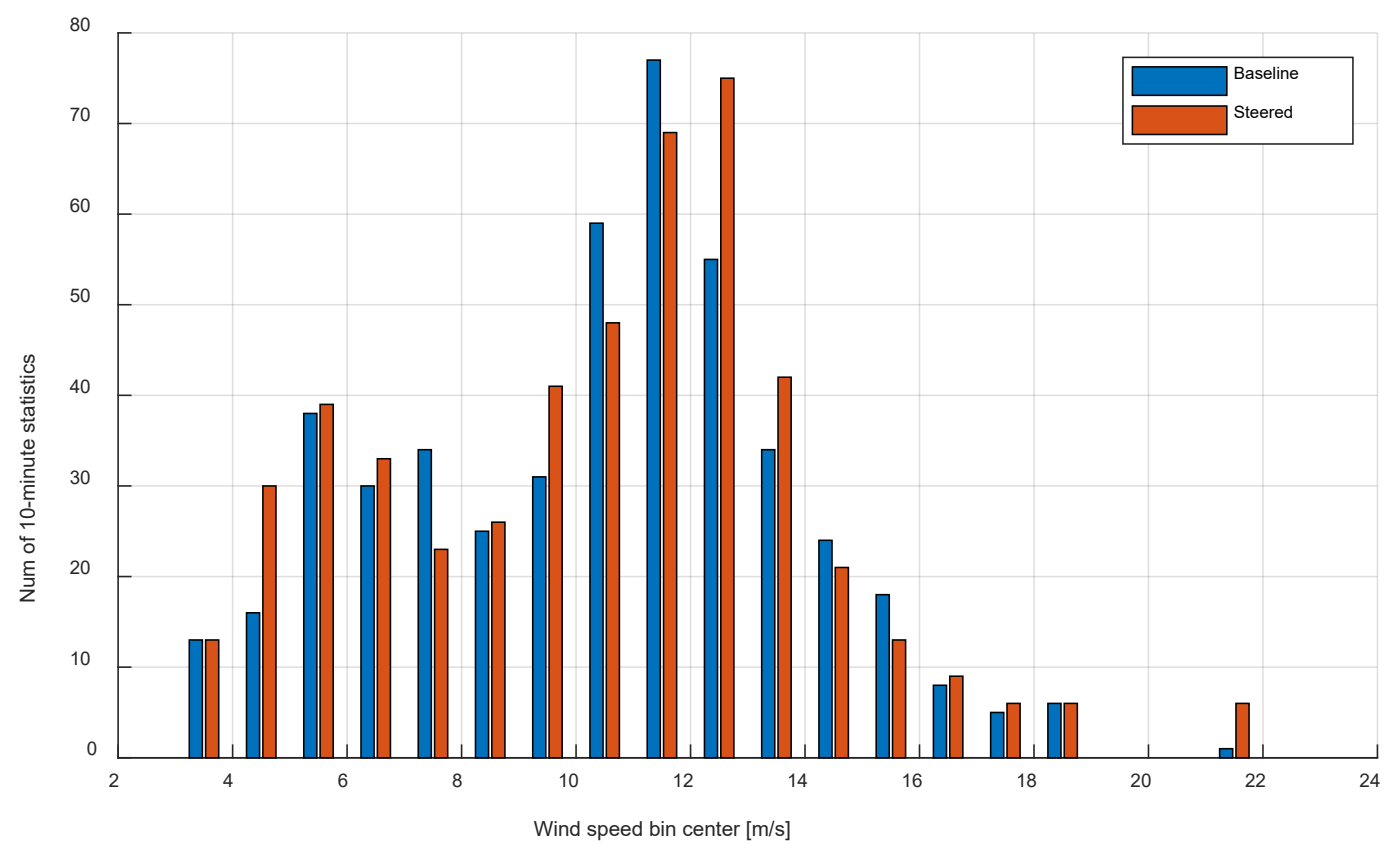

Figure 4. Comparison of histograms of 10-minute statistics per wind speed bin for the baseline and wake steered data sets

To arrive at the valid data in the two databases, the following filters were applied:

1. Only $50-\mathrm{Hz}$ data rate files of 600 seconds were used.

2. Data were limited to T2 mean wind direction values within the measurement sector of 320 to 350 relative to true north. This limitation was governed by the wake steering controller schedule presented in Figure 2.

3. Data were removed when the T3 DAS was not functioning normally.

4. Data were removed when any instrumentation used in the experiment was not functioning normally.

5. Data were removed when both $\mathrm{T} 2$ and $\mathrm{T} 3$ were not in a normal power production state as indicated by the turbine SCADA status signals and filtering for derated turbine operation.

6. Data were removed when $\mathrm{T} 3$ power production was below $0 \mathrm{~kW}$.

For the wind speed range of interest, where wake steering is applicable (approximately $3 \mathrm{~m} / \mathrm{s}$ to $14 \mathrm{~m} / \mathrm{s}$ ), sufficient data were collected allowing for a statistically meaningful analysis.

According to Figure 4 most wind speed bins exceed 20 valid data files for each database: the minimum requirement according to the Standard. Figure 5 provides the baseline capture matrix illustrating a total of 80.167 hours of valid data collected during the experiment. Figure 6 provides the wake steered capture matrix, which contained a total of 84.167 hours of valid data. 


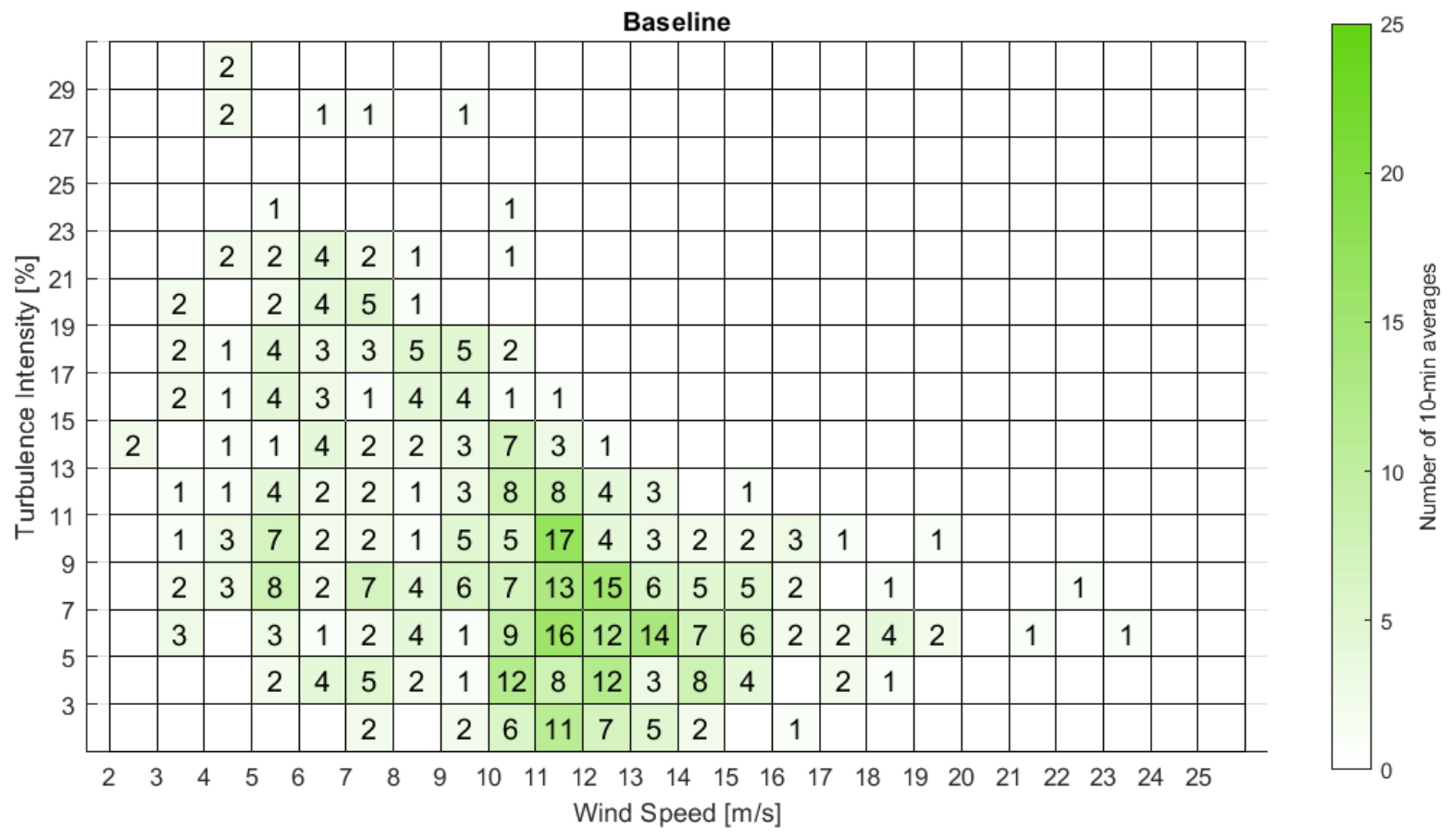

Figure 5. Capture matrix for baseline database

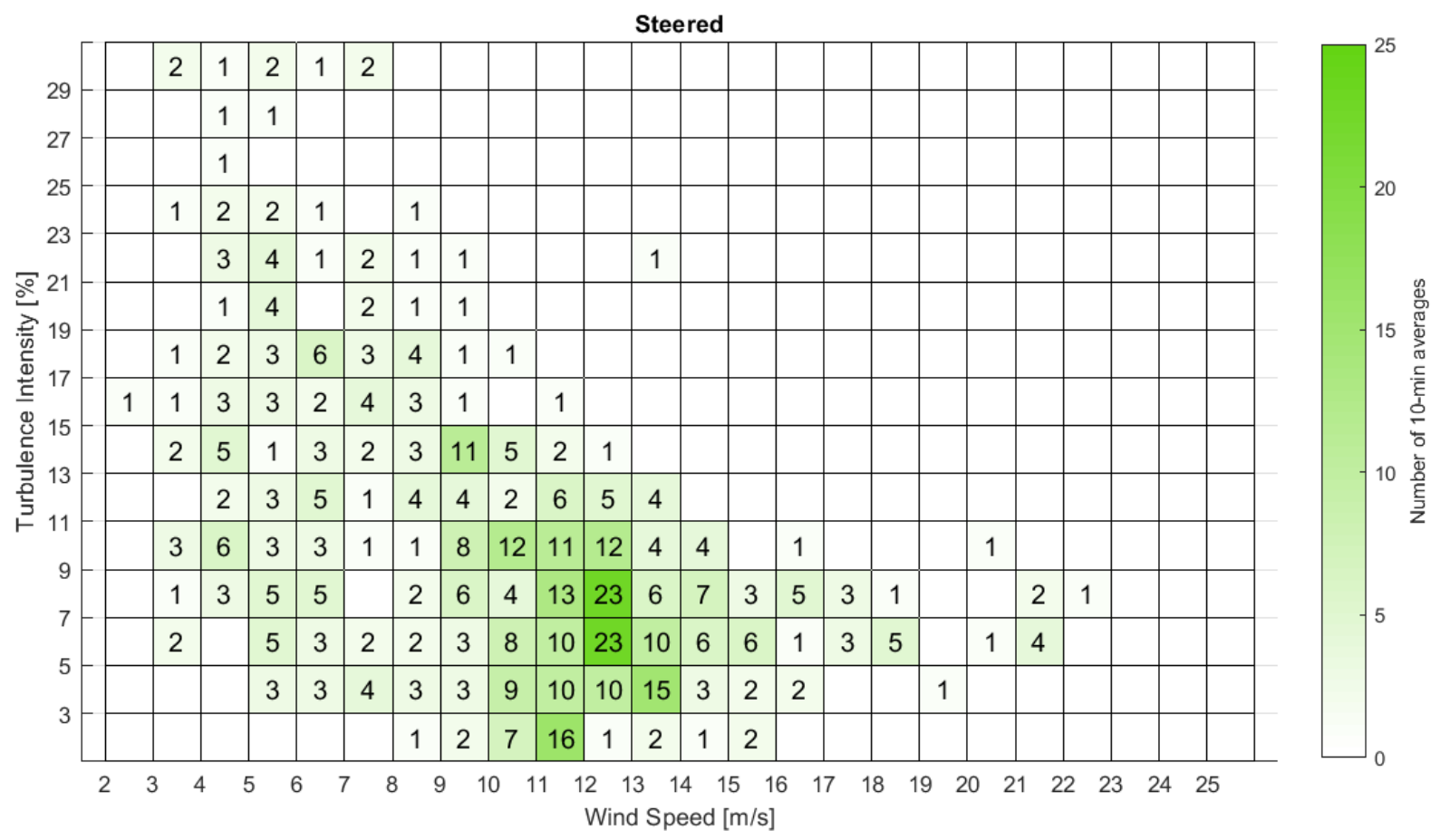

Figure 6. Capture matrix for wake steered database

A third database was created to account for the different yaw offset angles. It was directly derived from the steered data set by filtering the collected steered valid data into yaw offset bins with a range of $1.25^{\circ}-21.25^{\circ}$ and bin width of $2.5^{\circ}$ primarily based on the yaw offset controller 
schedule found in Figure 2. The modified capture matrix in Figure 7 provides the resulting number of valid data after this filter was applied. Note that this custom database does not account for differences in turbulence intensity bins. This capture matrix provides insights into the wind speed and yaw offset distribution during times of wake steering control; however, this was not investigated further in the context of the loads response for $\mathrm{T} 3$.
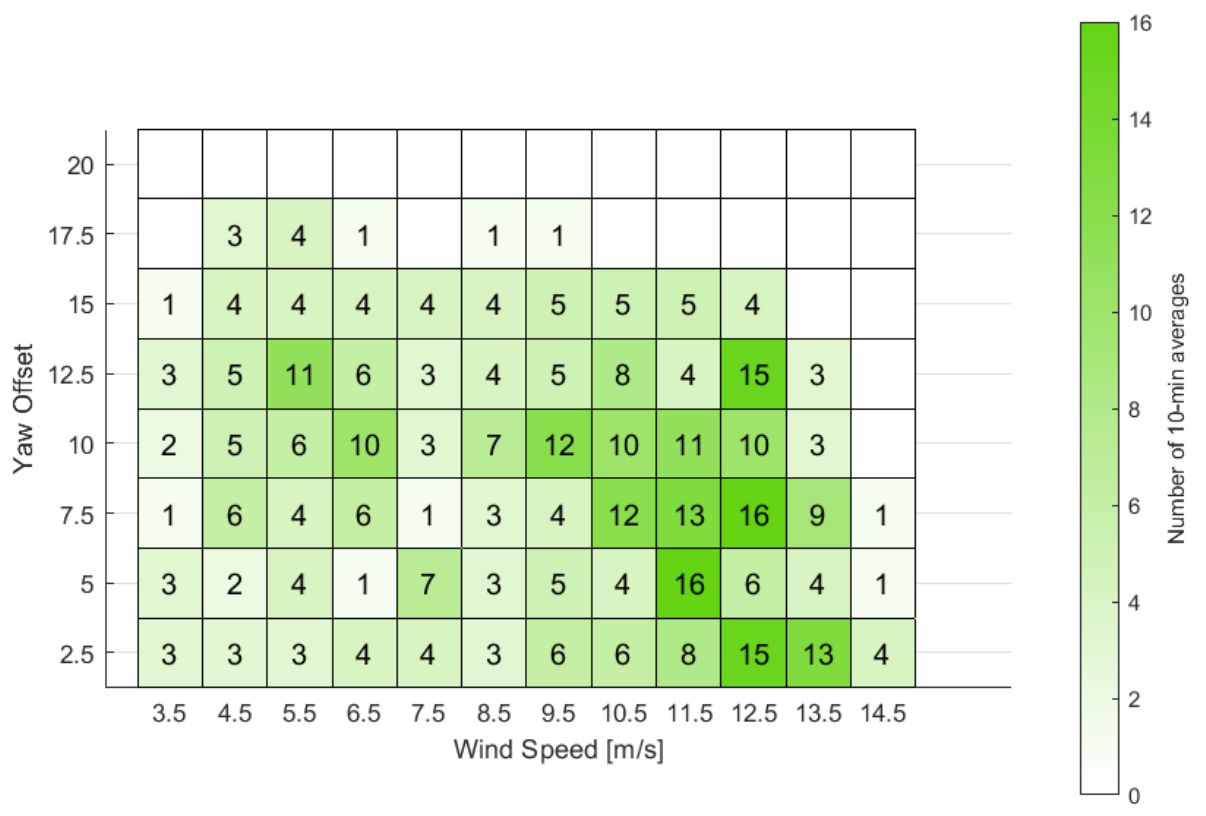

Figure 7. Capture matrix representing different yaw offset angles derived from wake steered database 


\section{Data Analysis Procedure}

This section describes the data analysis process and tools used for loads and fatigue.

All valid data files were processed to calculate scaled loads in engineering units and 10-minute statistics to include means, maxima, minima, and standard deviations. The statistical results are presented in Section 7.

The main shaft operating loads were calculated into nonrotating shaft loads in the nacelle coordinate system using the rotor azimuth position. In this case, the rotor tilt moment $(M y)$ and rotor yaw moment $(\mathrm{Mz})$ are reported. The tower loads are calculated into the nacelle reference coordinate system and the fore-aft and side-to-side moments using yaw position. These results can be found in Section 7. See Appendix A for the coordinate systems that describe these load paths.

The method of bins was used, as per the Standard, to determine bin averages and bin standard deviations for operating loads and damage equivalent loads (DELs). All data were binned using wind speed with $1-\mathrm{m} / \mathrm{s}$ bin widths starting at $3 \mathrm{~m} / \mathrm{s}$ and ending at $15 \mathrm{~m} / \mathrm{s}$. The upper range was set to be near rated wind speed $(V r=14 \mathrm{~m} / \mathrm{s})$, limiting the analysis to the wind speed range where wake steering controls are most applicable: in Region 2 up to $12 \mathrm{~m} / \mathrm{s}$.

The DELs for the operating moments were calculated in accordance with the Standard using material slopes $(m)$ typical for the component and without Goodman correction. The material slope value was 4 for the tower and main shaft loads, and a value of 10 was used for the blades. An exponent of 4 is typical for steel, whereas an exponent of 10 is more common for fiberglass and other similar composites used in blades. The DEL calculations were carried out using MLife [5], a MATLAB-based postprocessing tool developed by NREL to analyze wind turbine data, and aeroelastic/dynamics simulations. MLife uses the one-pass cycle-counting method of Downing and Socie for fatigue cycle counting. For the analysis in this report, a cycle count of 0.5 was assigned to unclosed cycles. Because wake steering controls are used for very specific operating and inflow conditions, a typical wind speed distribution (Rayleigh) for lifetime fatigue calculation is not applicable. As a result, only short-term DELs were determined using 100 loadrange bins. 


\section{Results}

The results presented here illustrate the differences in the mechanical loads of T3 between the baseline and wake-steered case. Only the wind speeds ranges between $4 \mathrm{~m} / \mathrm{s}$ and $15 \mathrm{~m} / \mathrm{s}$ are provided to illustrate the influence of wake steering (see Figure 2).

\subsection{Turbulence Intensity}

Turbulence intensity (TI) results are provided in the following two figures. The TI scatter reported in Figure 8 provides a comparison of the baseline and steered databases derived by the filters discussed in Section 5. Figure 9 shows the turbulence intensity binned averages for the two databases. The error bars in Figure 9 represent $+/$ - one standard deviation $(\sigma)$ of the TI data within each wind speed bin; the error bar length is $2 \sigma$. Research has indicated that turbulence has a major influence on the fatigue loading of wind turbine components (see recent examples in [1] and [6]). The results presented here show that the differences in TI between the baseline and steered databases are minimal, but a superposition of turbulence cannot be discounted.

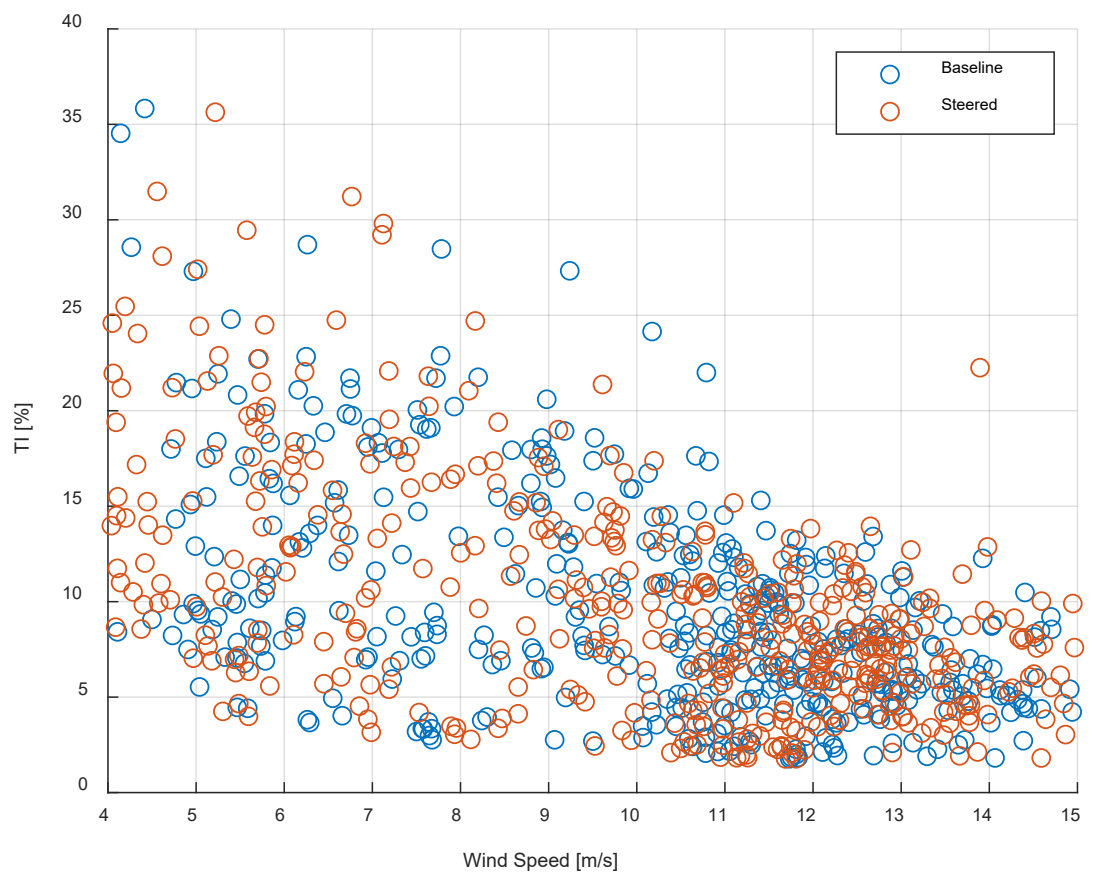

Figure 8. Comparison of turbulence intensity 10-minute statistics 


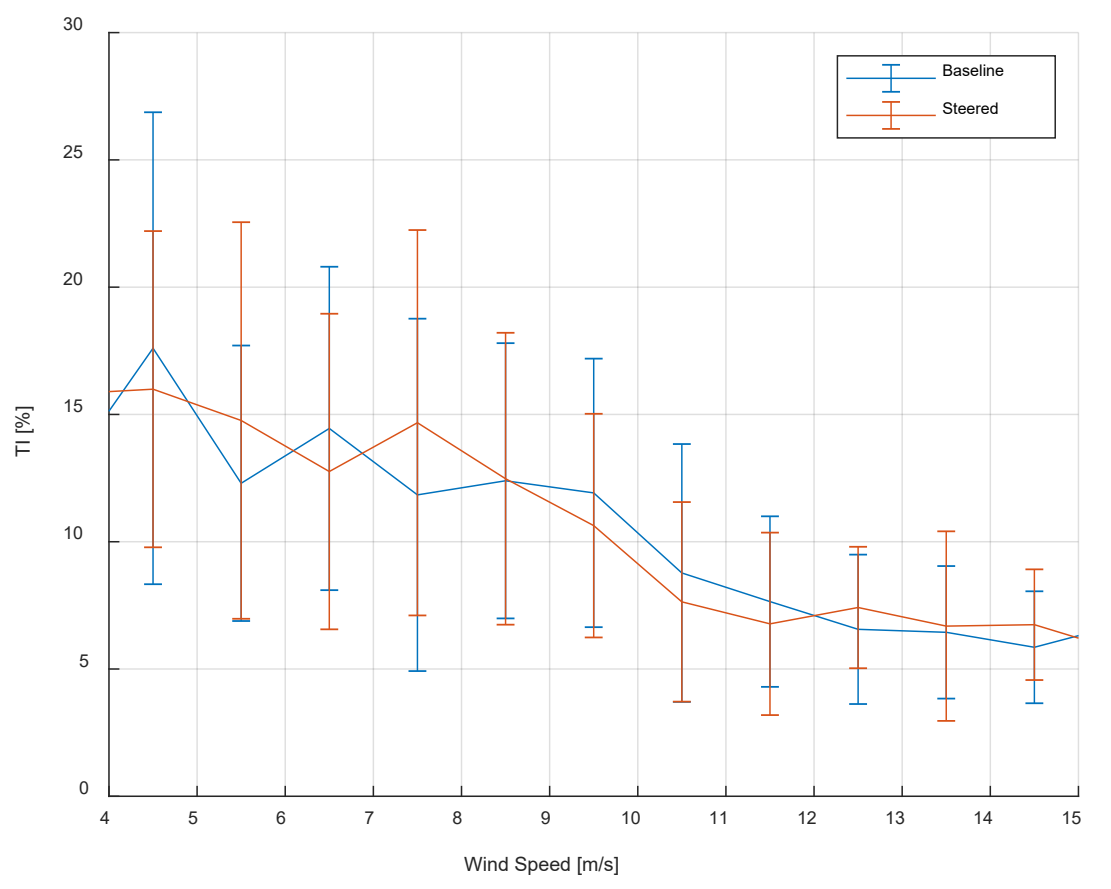

Figure 9. Comparison of binned statistics for baseline and steered turbulence intensity

Error bars are $+/-1 \sigma$.

\subsection{Binned Statistics}

This section presents the binned statistics of each load variable. For each statistic, the bin means and standard deviations were determined.

Each load variable is normalized by its baseline absolute maximum mean value. Table 3 summarizes the corresponding wind speed at which this normalization value occurs. See Appendix B for corresponding statistical scatter plots normalized by the same values used in these binned statistics.

In some instances, this normalization might be difficult to interpret. For instance, the main shaft pitching moment $\left(M_{y}\right)$ and the tower top fore-aft bending moments both achieve a maximum mean load at low wind speeds. This is a result of gravity loading created by the rotor overhang mass, which is then offset by the rotor thrust load as wind speed increases causing the load response to trend toward zero. This variable normalization method is applied, rather than choosing a specific wind speed, to keep values in a range where they would be easier to comprehend, especially for loads where the mean is close to zero. Normalization where values trend to zero or cross zero can have an exponential effect that is disingenuous to the nonnormalized response.

For added clarity to the blade flapwise response shown in Figure 10, a negative moment is a result of load application in the downwind direction; this is an artifact of the strain gage installation and was not manipulated in postprocessing. The coordinate systems provided in Appendix A demonstrate the sign conventions used for all loads components. 
Table 3. Summary of Wind Speeds Corresponding to Baseline Absolute Maximum Mean Value of Load Variable Used for Normalization of Binned Statistics

\begin{tabular}{|l|l|}
\hline Load Variable & Wind Speed $(\mathbf{m} / \mathbf{s})$ \\
\hline Blade 1 root flapwise bending moment & 10.5 \\
\hline Blade 1 root edgewise bending moment & 10.5 \\
\hline Main shaft $M_{y}$ bending moment & 3.5 \\
\hline Main shaft $M_{z}$ bending moment & 8.5 \\
\hline Main shaft torque & 12.5 \\
\hline Tower top fore-aft bending moment & 3.5 \\
\hline Tower top side-side bending moment & 12.5 \\
\hline Tower top torque & 8.5 \\
\hline Tower base fore-aft bending moment & 10.5 \\
\hline Tower base side-side bending moment & 7.5 \\
\hline
\end{tabular}

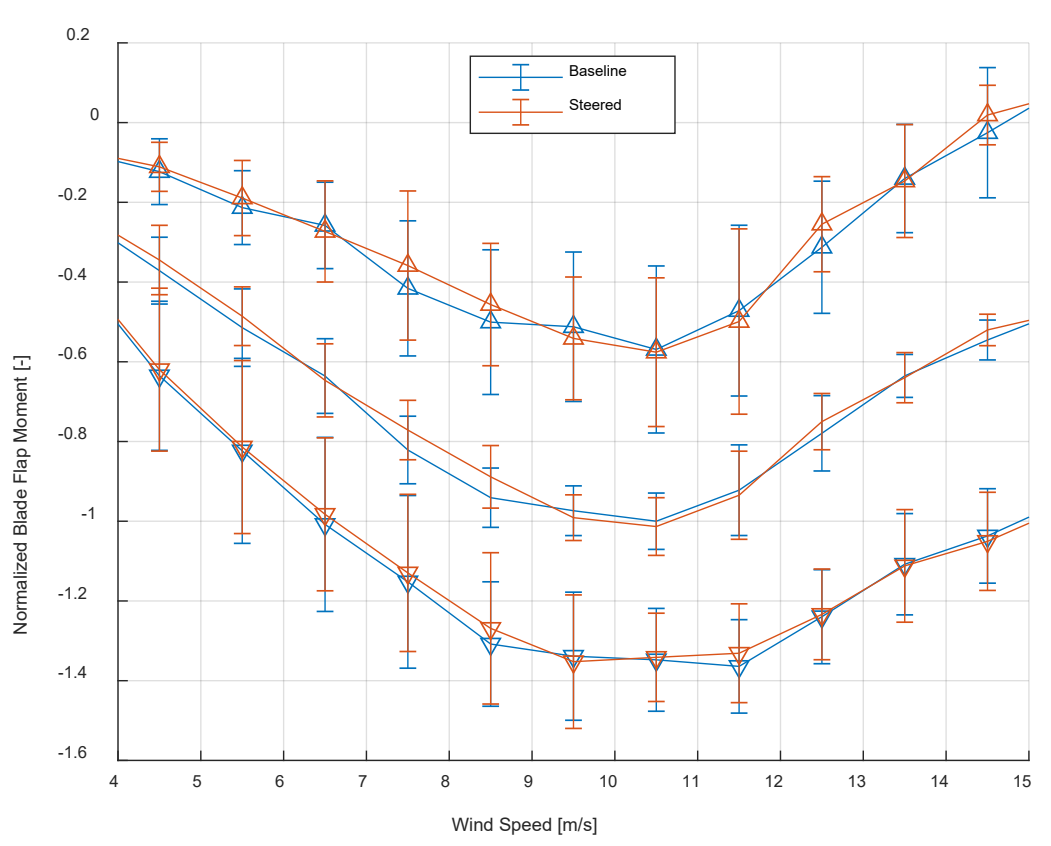

Figure 10. Comparison of binned statistics for normalized baseline and steered blade 1 root flapwise bending moments

$\mathbf{\Delta}=$ maxima, $-=$ means, $\boldsymbol{\nabla}=$ minima. Error bars are $+/-1 \sigma$. 


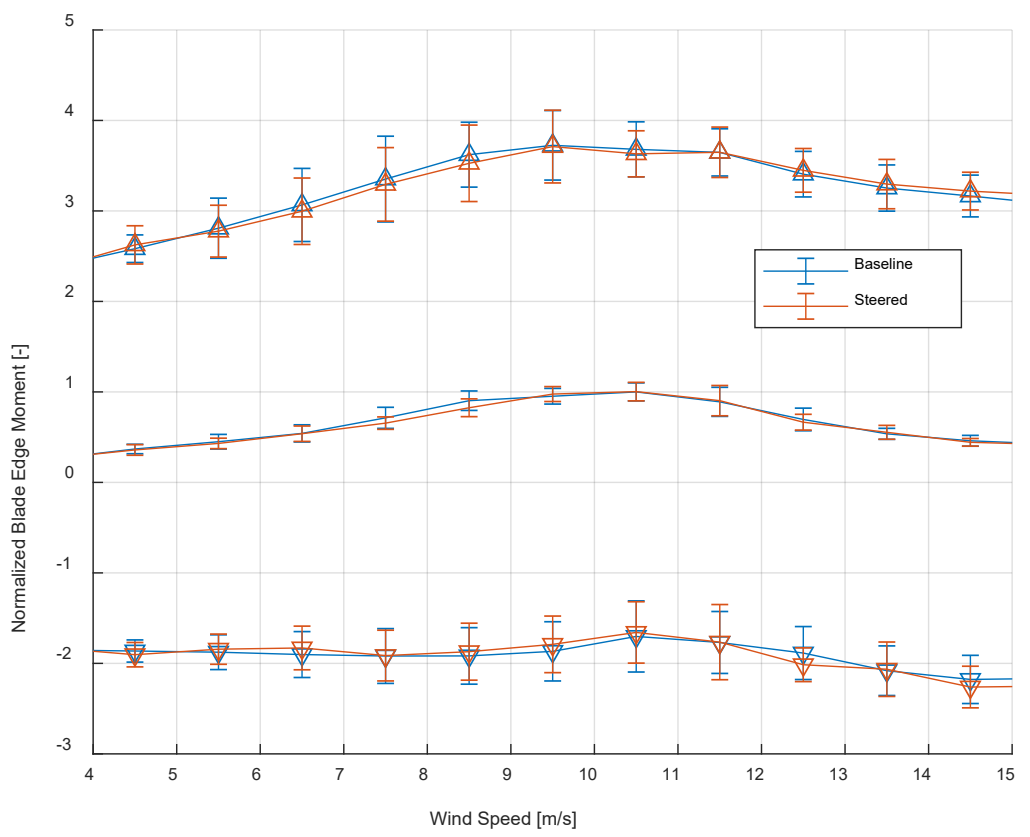

Figure 11. Comparison of normalized binned statistics for baseline and steered blade 1 edgewise bending moments

$\boldsymbol{\Delta}=$ maxima,$-=$ means, $\boldsymbol{\nabla}=$ minima. Error bars are $+/-1 \sigma$.

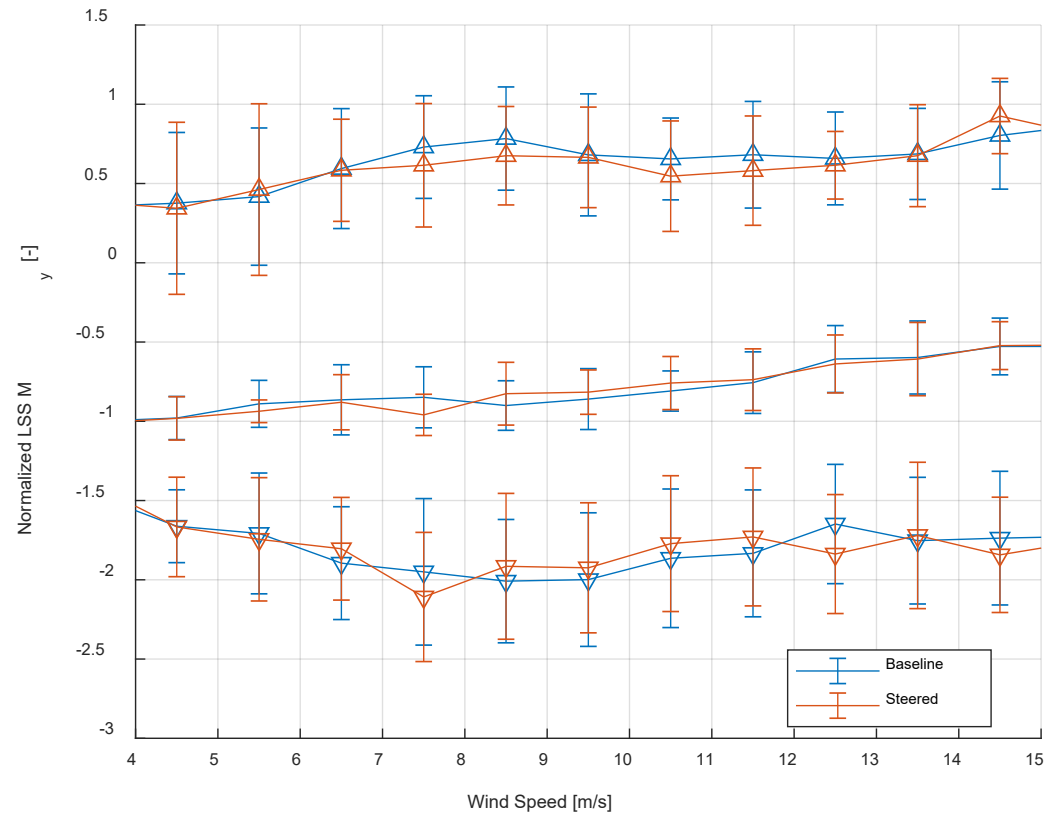

Figure 12. Comparison of normalized binned statistics for baseline and steered main shaft $M_{y}$ bending moments

$\boldsymbol{\Delta}=$ maxima,$-=$ means, $\boldsymbol{\nabla}=$ minima. Error bars are $+/-1 \sigma$. 


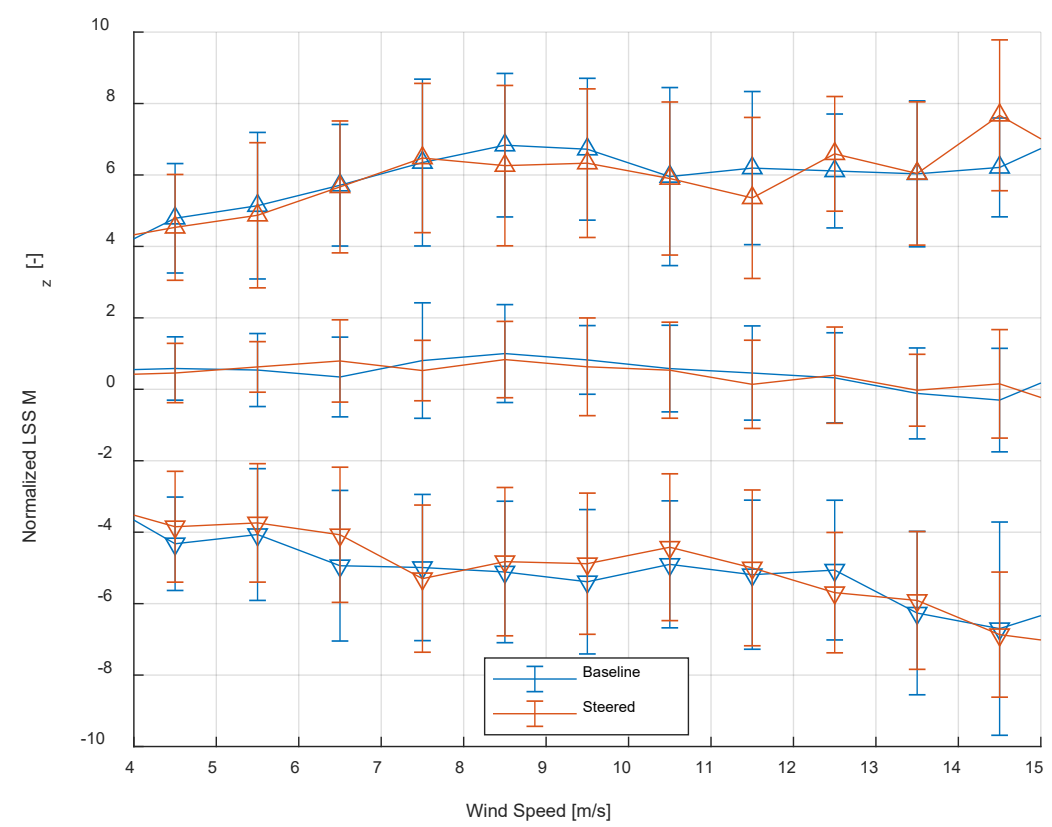

Figure 13. Comparison of normalized binned statistics for baseline and steered main shaft $M_{z}$ bending moments

$\boldsymbol{\Delta}=$ maxima,$-=$ means, $\boldsymbol{\nabla}=$ minima. Error bars are $+/-1 \sigma$.

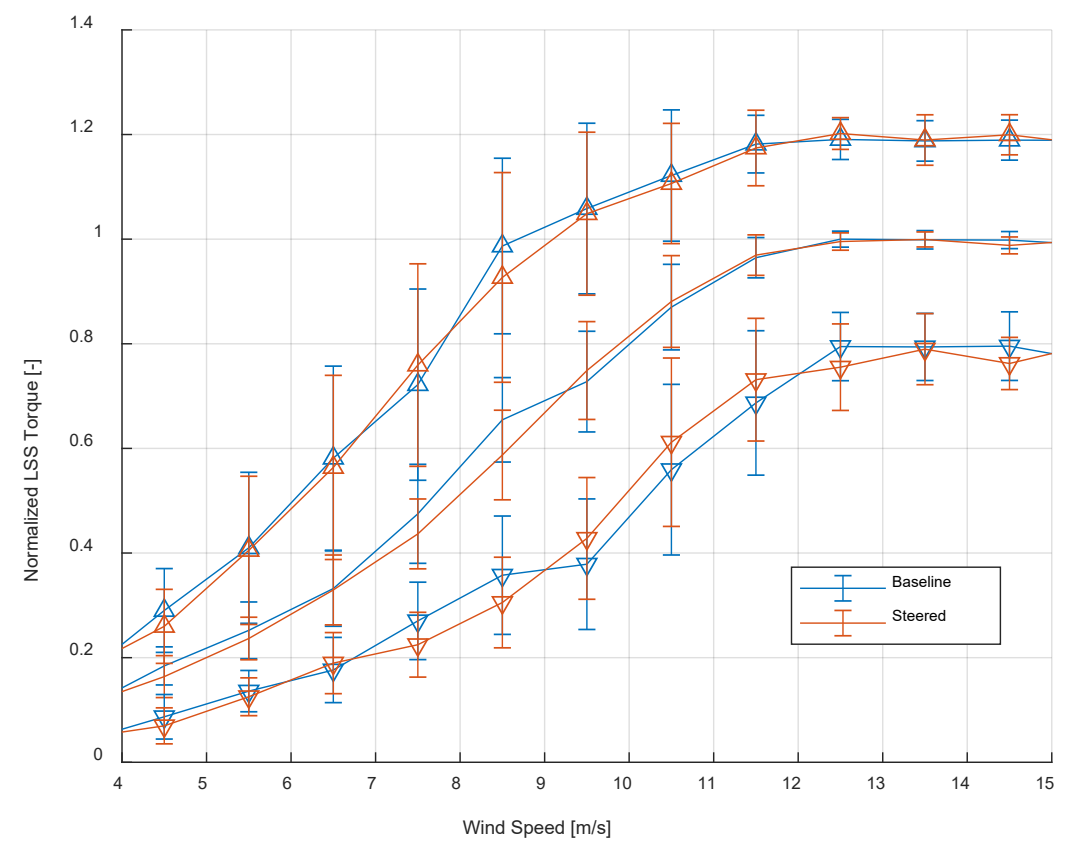

Figure 14. Comparison of normalized binned statistics for baseline and steered main shaft torque response

$\boldsymbol{\Delta}=$ maxima,$-=$ means, $\boldsymbol{\nabla}=$ minima. Error bars are $+/-1 \sigma$. 


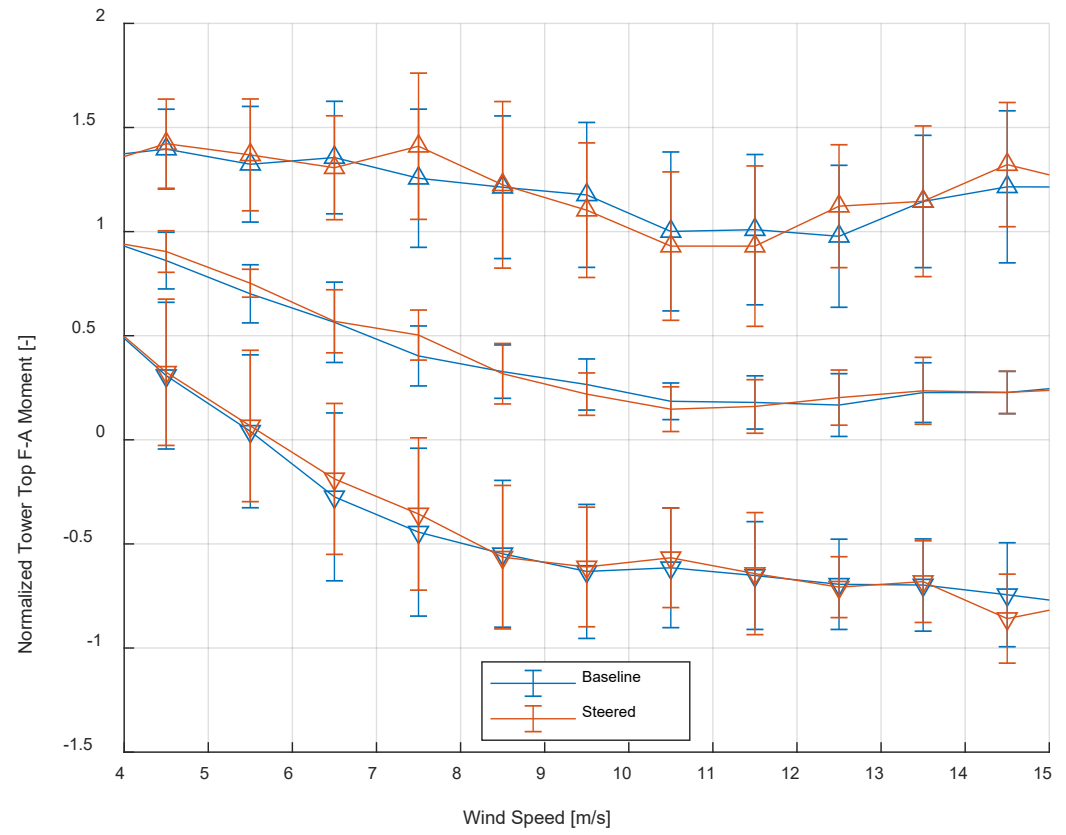

Figure 15. Comparison of normalized binned statistics for baseline and steered tower top fore-aft bending moments

$\boldsymbol{\Delta}=$ maxima,$-=$ means, $\boldsymbol{\nabla}=$ minima. Error bars are $+/-1 \sigma$.

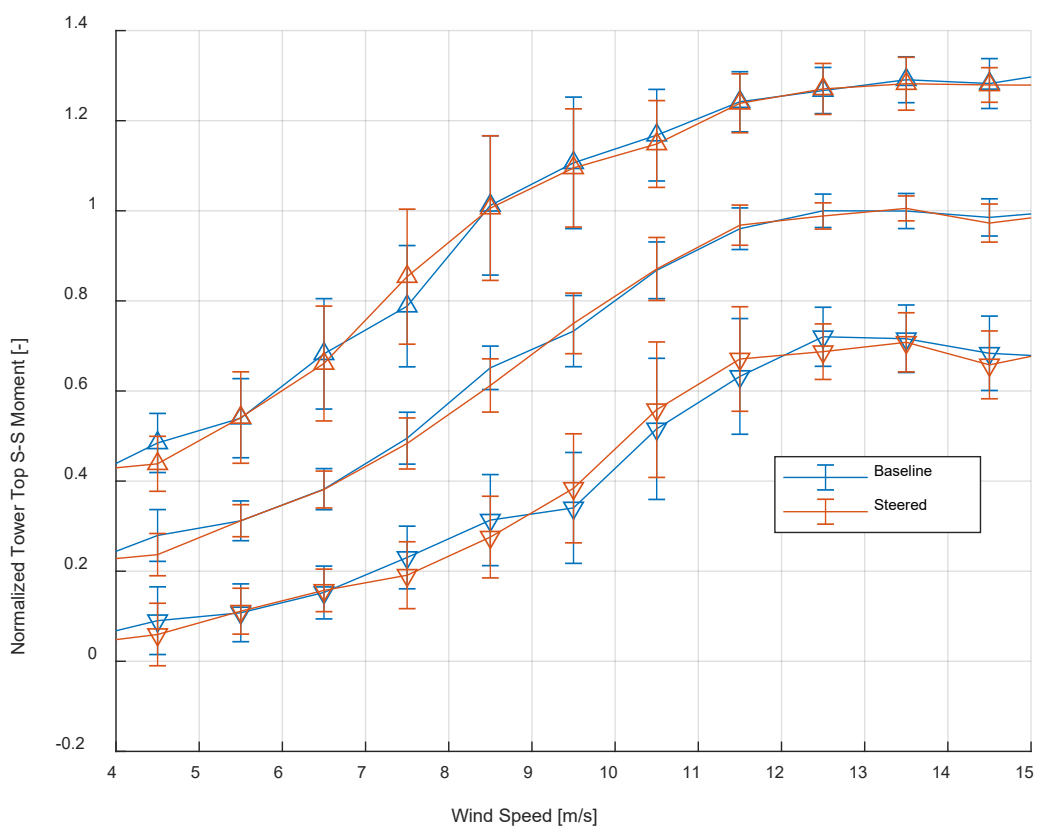

Figure 16. Comparison of normalized binned statistics for baseline and steered tower top sideside bending moments

$\boldsymbol{\Delta}$ = maxima,$-=$ means, $\boldsymbol{\nabla}=$ minima. Error bars are $+/-1 \sigma$. 


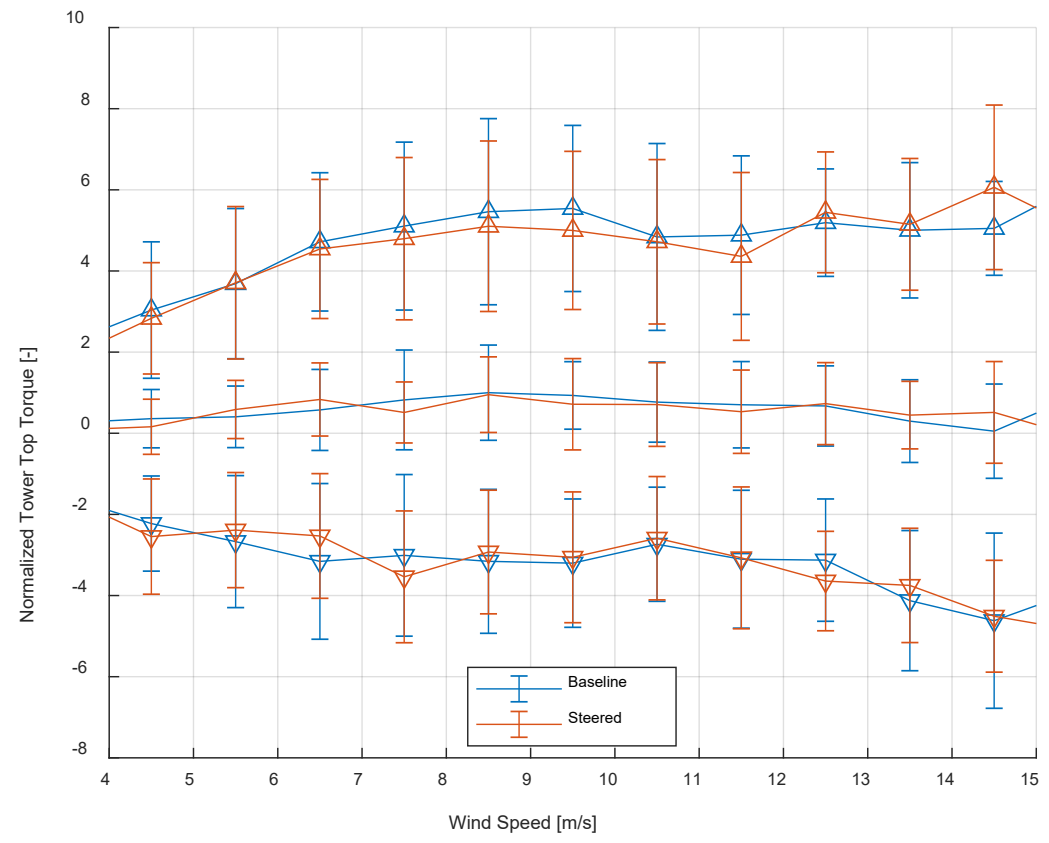

Figure 17. Comparison of normalized binned statistics for baseline and steered tower top torque response

$\boldsymbol{\Delta}=$ maxima,$-=$ means, $\boldsymbol{\nabla}=$ minima. Error bars are $+/-1 \sigma$.

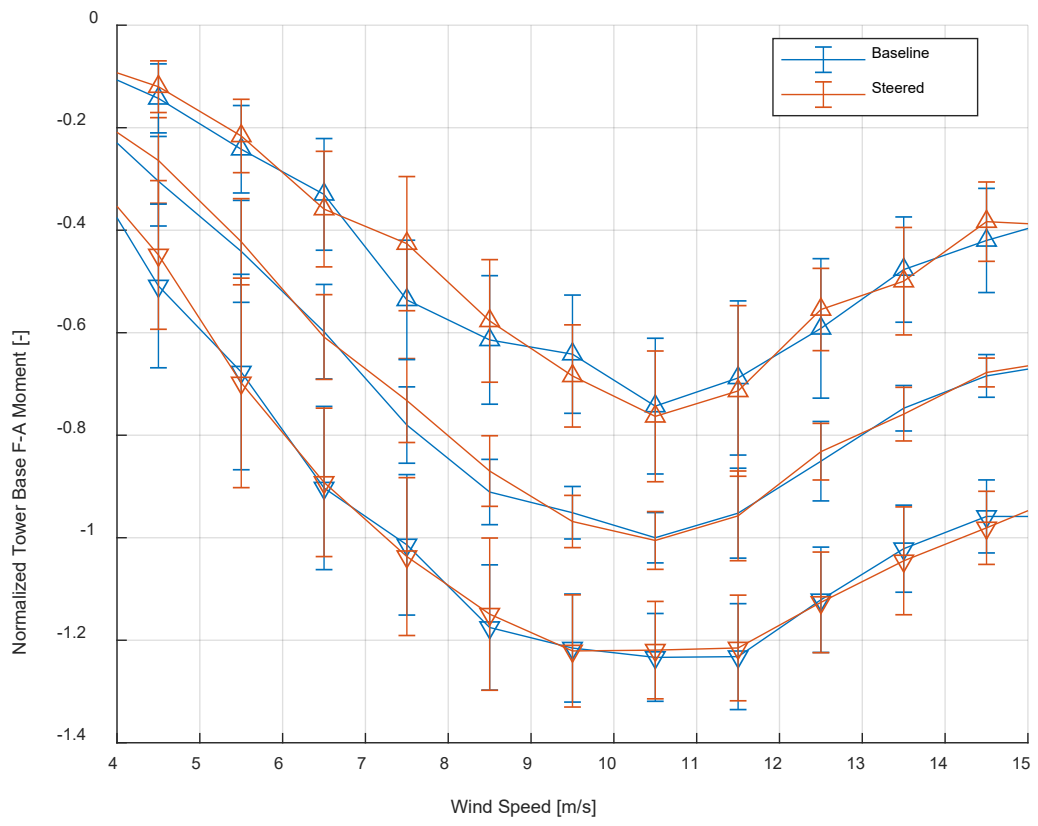

Figure 18. Comparison of normalized binned statistics for baseline and steered tower base fore-aft bending moments

$\boldsymbol{\Delta}$ = maxima, $-=$ means, $\boldsymbol{\nabla}=$ minima. Error bars are $+/-1 \sigma$. 


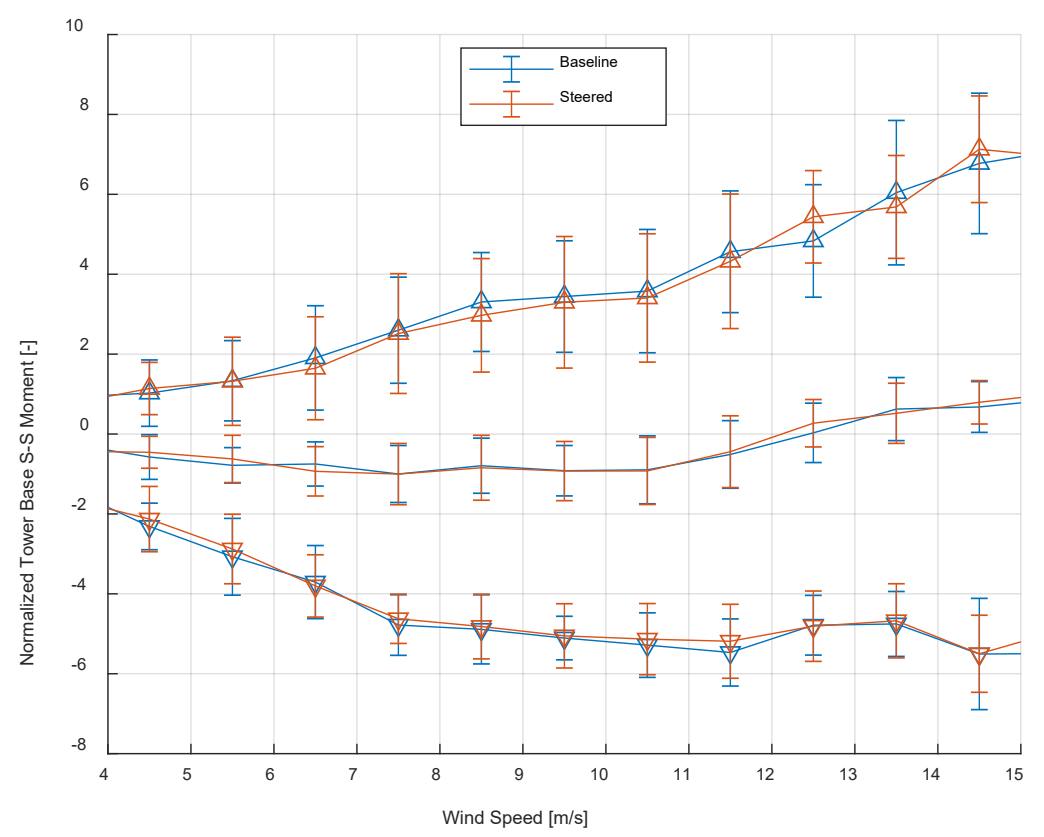

Figure 19. Comparison of normalized binned statistics for baseline and steered tower base sideside bending moments

$\boldsymbol{\Delta}=$ maxima,$-=$ means, $\boldsymbol{\nabla}=$ minima. Error bars are $+/-1 \sigma$.

\subsection{Binned Damage Equivalent Loads}

This section presents the results of the binned short-term (10-minute) damage equivalent loads. Like the binned statistics, each load variable is normalized by its baseline absolute maximum value, which can occur at different wind speeds. Table 4 summarizes the corresponding wind speed at which this normalization value occurs. See Appendix C for corresponding DEL scatterplots normalized by the same values used with the binned DEL figures.

Table 4. Summary of Wind Speed Corresponding to Baseline Absolute Maximum Value of Load Variable Used for Normalization of Binned DELs

\begin{tabular}{|l|l|}
\hline Load Variable & Wind Speed $(\mathrm{m} / \mathbf{s})$ \\
\hline Blade 1 root flapwise bending moment & 14.5 \\
\hline Blade 1 root edgewise bending moment & 9.5 \\
\hline Main shaft $M_{y}$ bending moment & 14.5 \\
\hline Main shaft $M_{z}$ bending moment & 14.5 \\
\hline Main shaft torque & 9.5 \\
\hline Tower top fore-aft bending moment & 14.5 \\
\hline Tower top side-side bending moment & 14.5 \\
\hline Tower top torque & 14.5 \\
\hline Tower base fore-aft bending moment & 14.5 \\
\hline Tower base side-side bending moment & 14.5 \\
\hline
\end{tabular}




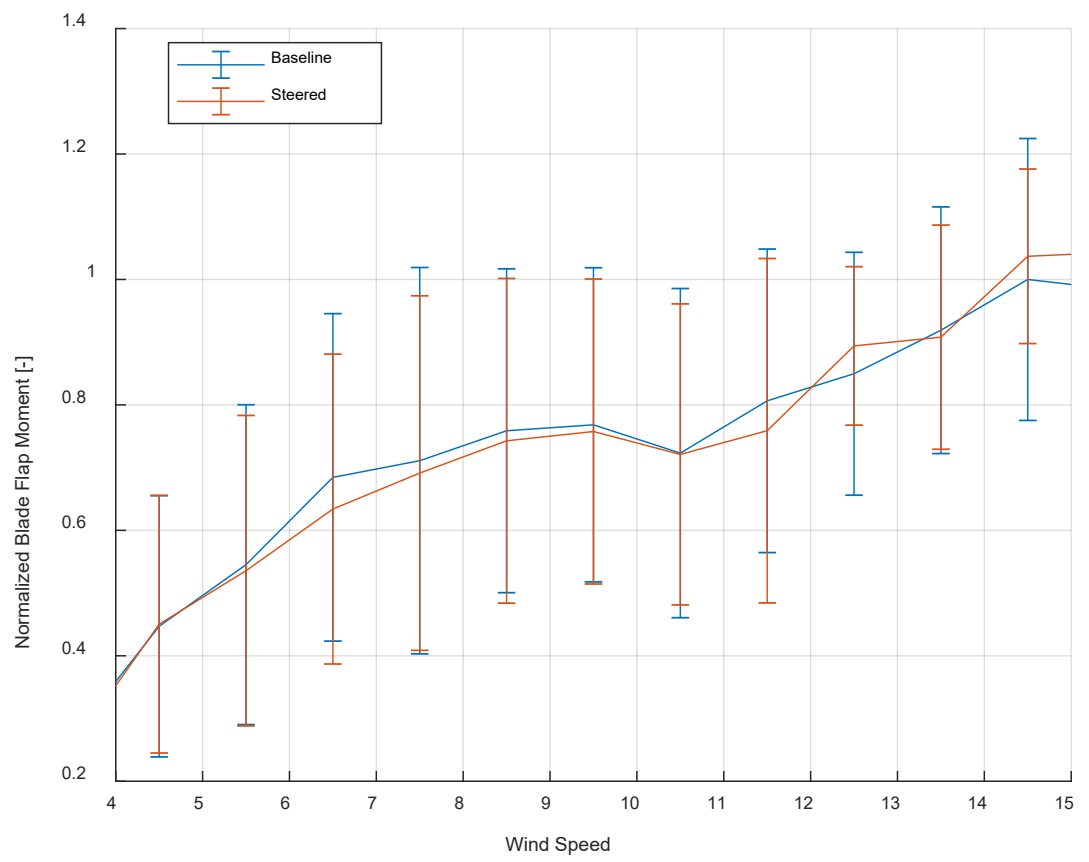

Figure 20. Comparison of normalized blade 1 root flapwise binned short-term (10-minute) damage equivalent loads

Error bars are $+/-1 \sigma$.

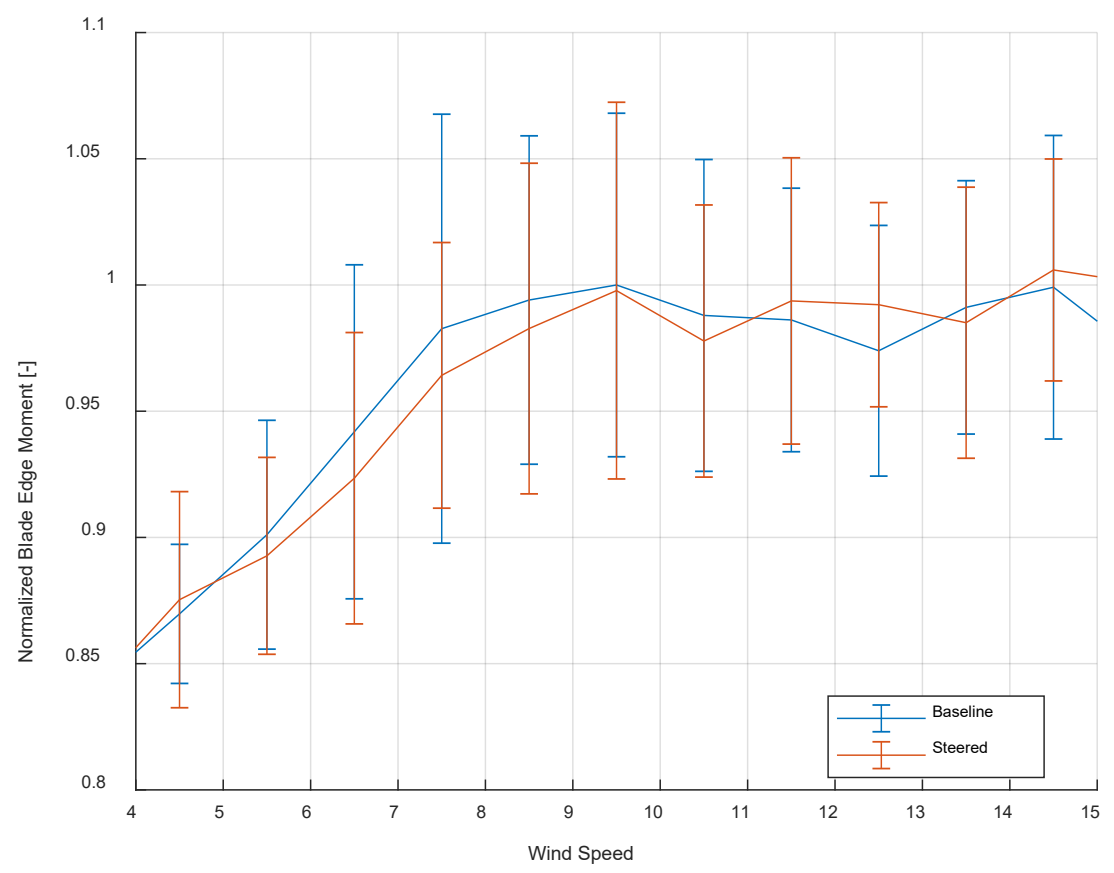

Figure 21. Comparison of normalized blade 1 root edgewise binned short-term (10-minute) damage equivalent loads

Error bars are $+/-1 \sigma$. 


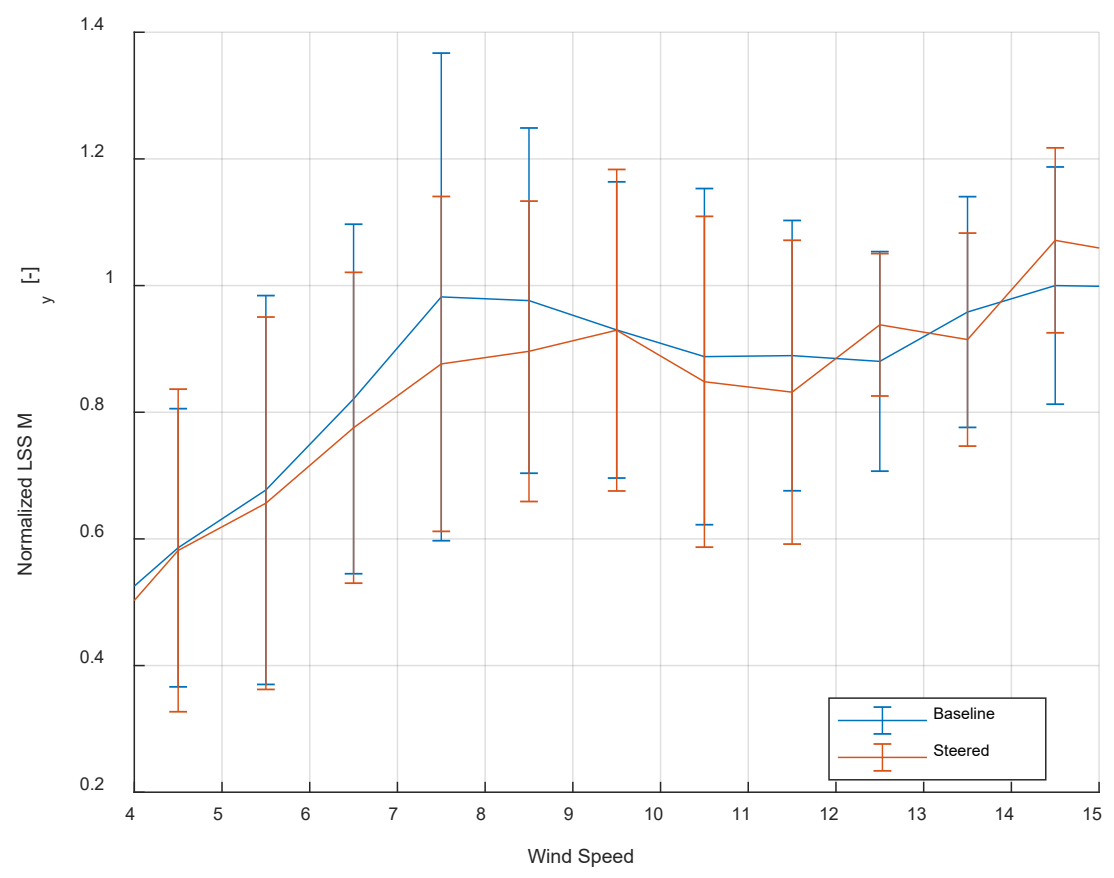

Figure 22. Comparison of normalized main shaft $M_{y}$ binned short-term (10-minute) damage equivalent loads

Error bars are $+/-1 \sigma$.

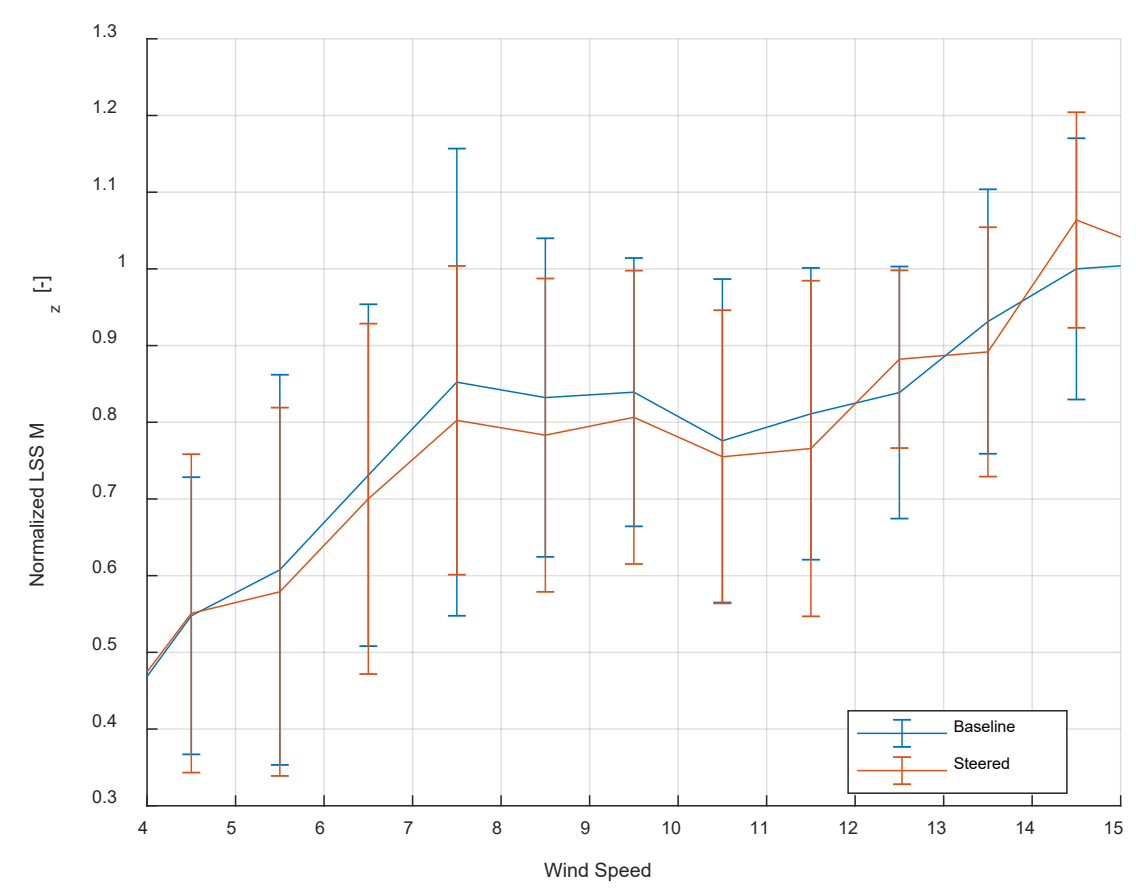

Figure 23. Comparison of normalized main shaft $M_{z}$ binned short-term (10-minute) damage equivalent loads

Error bars are $+/-1 \sigma$. 


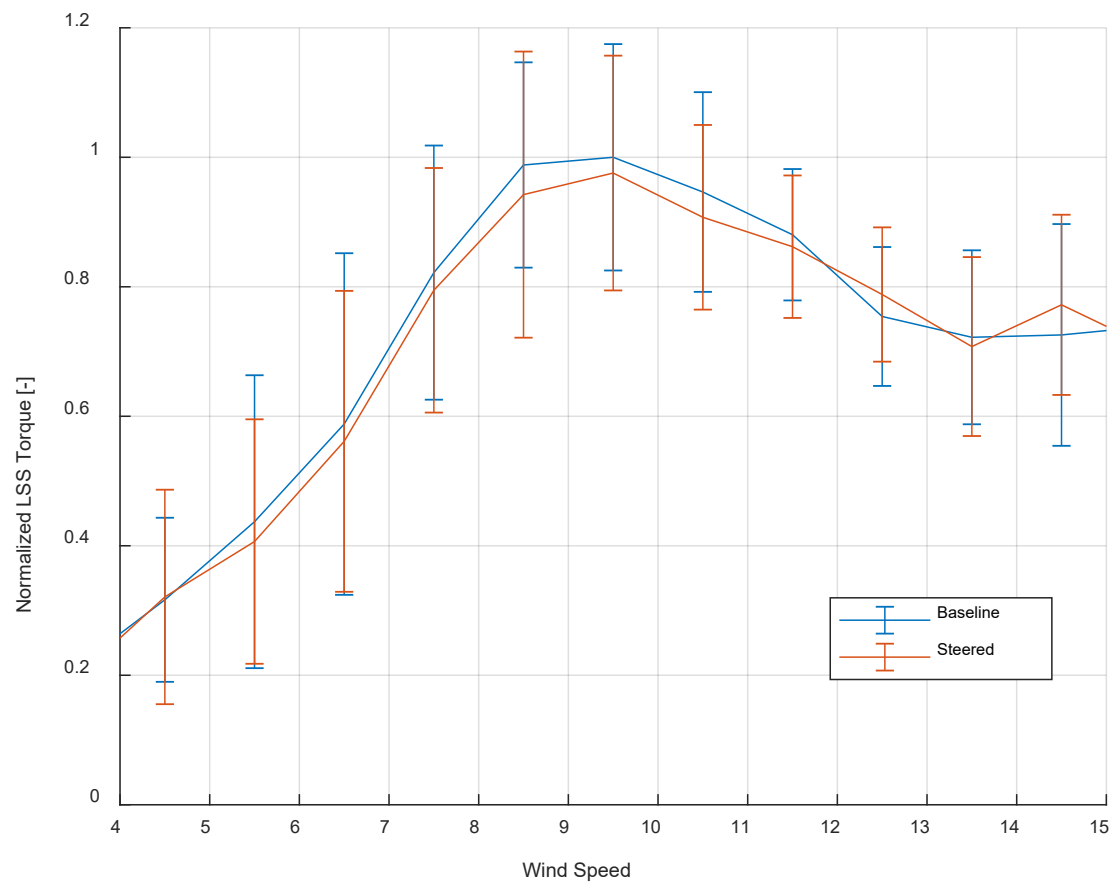

Figure 24. Comparison of normalized main shaft torque binned short-term (10-minute) damage equivalent loads

Error bars are $+/-1 \sigma$.

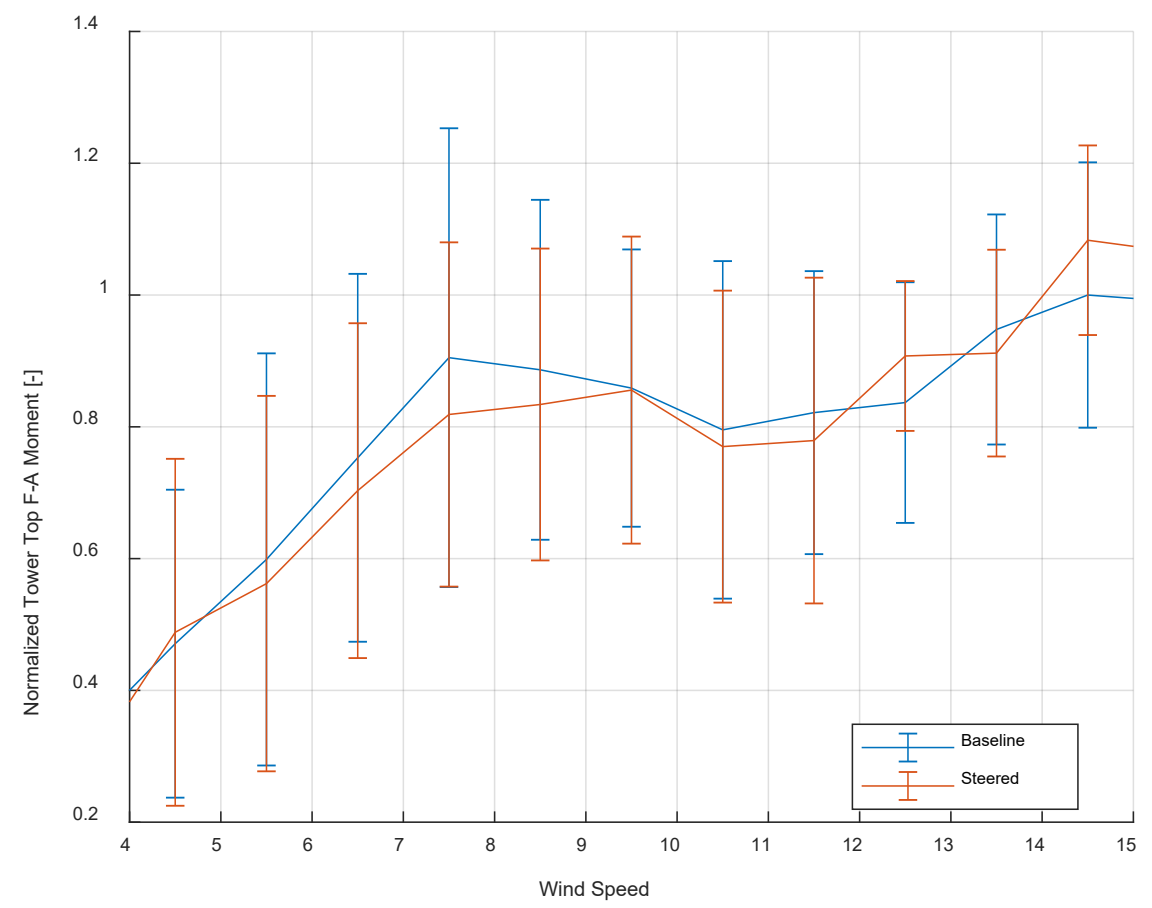

Figure 25. Comparison of normalized tower top fore-aft binned short-term (10-minute) damage equivalent loads 


\section{Error bars are $+/-1 \sigma$.}

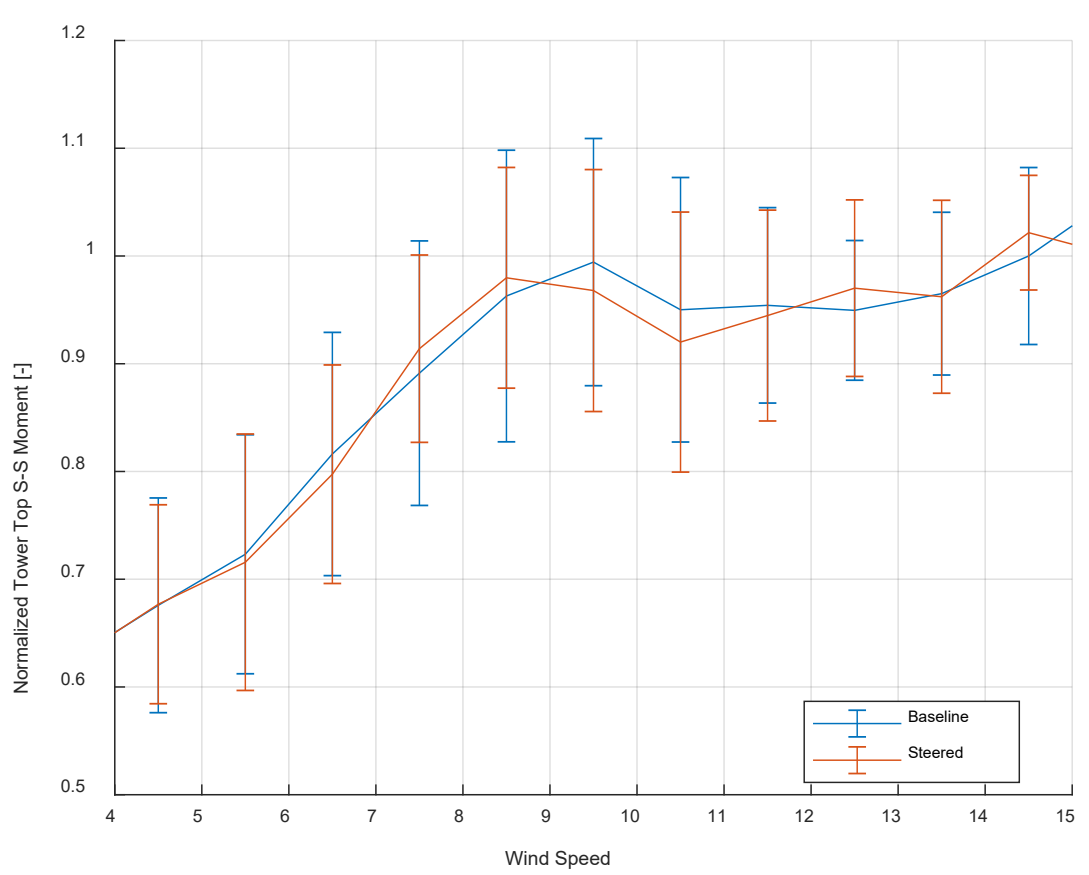

Figure 26. Comparison of normalized tower top side-side binned short-term (10-minute) damage equivalent loads

Error bars are $+/-1 \sigma$.

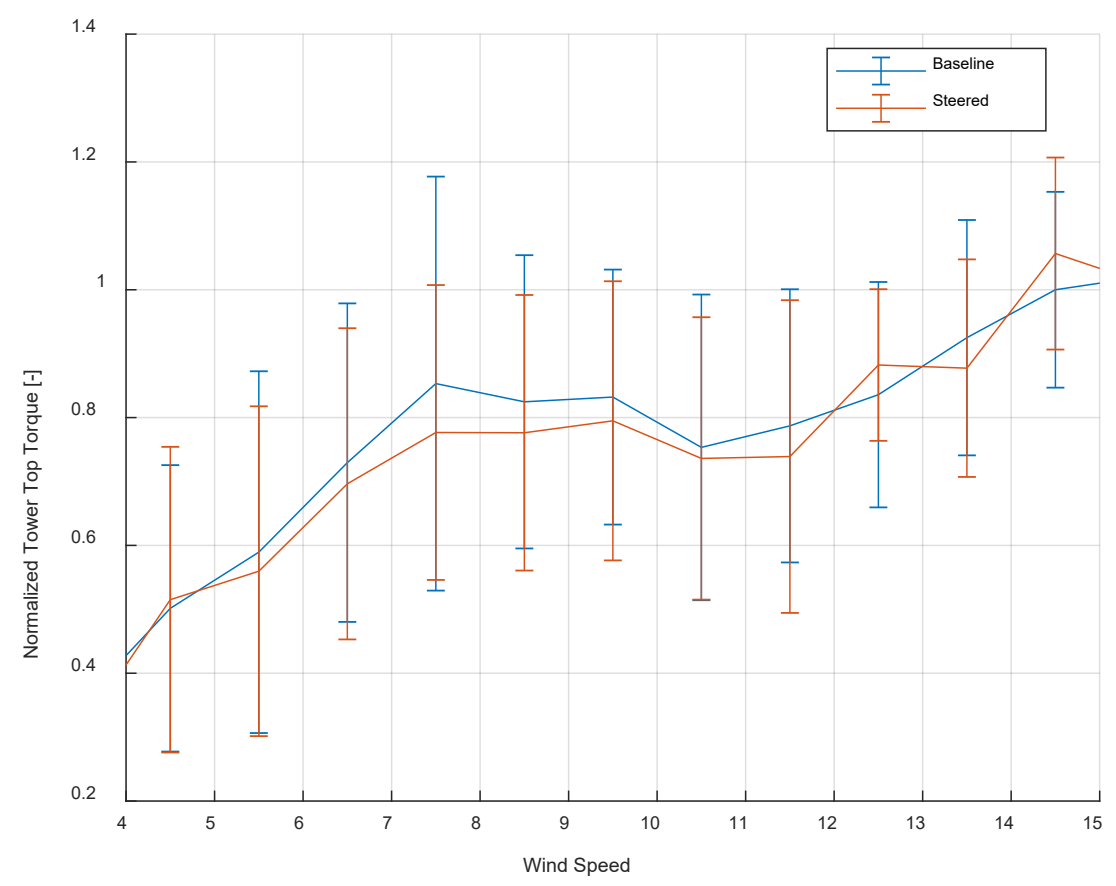

Figure 27. Comparison of normalized tower top torque binned short-term (10-minute) damage equivalent loads 


\section{Error bars are $+/-1 \sigma$.}

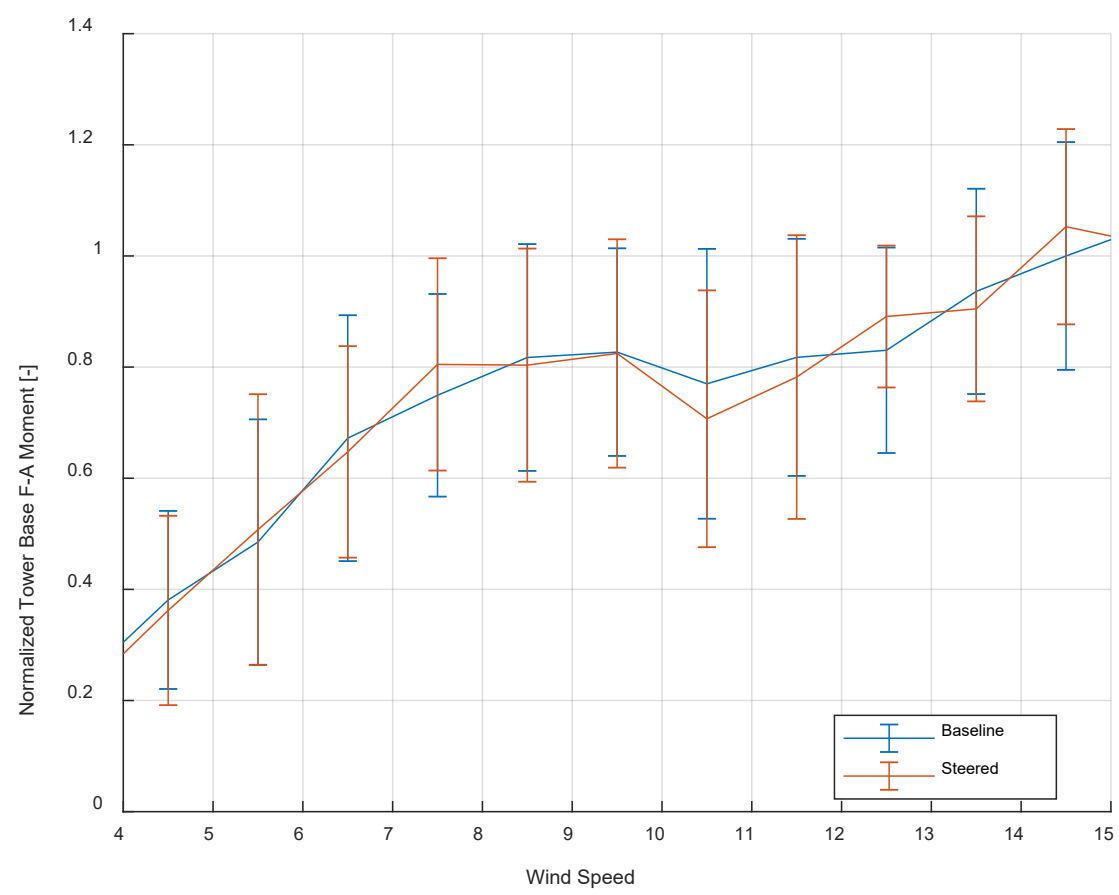

Figure 28. Comparison of normalized tower base fore-aft binned short-term (10-minute) damage equivalent loads

Error bars are $+/-1 \sigma$. 


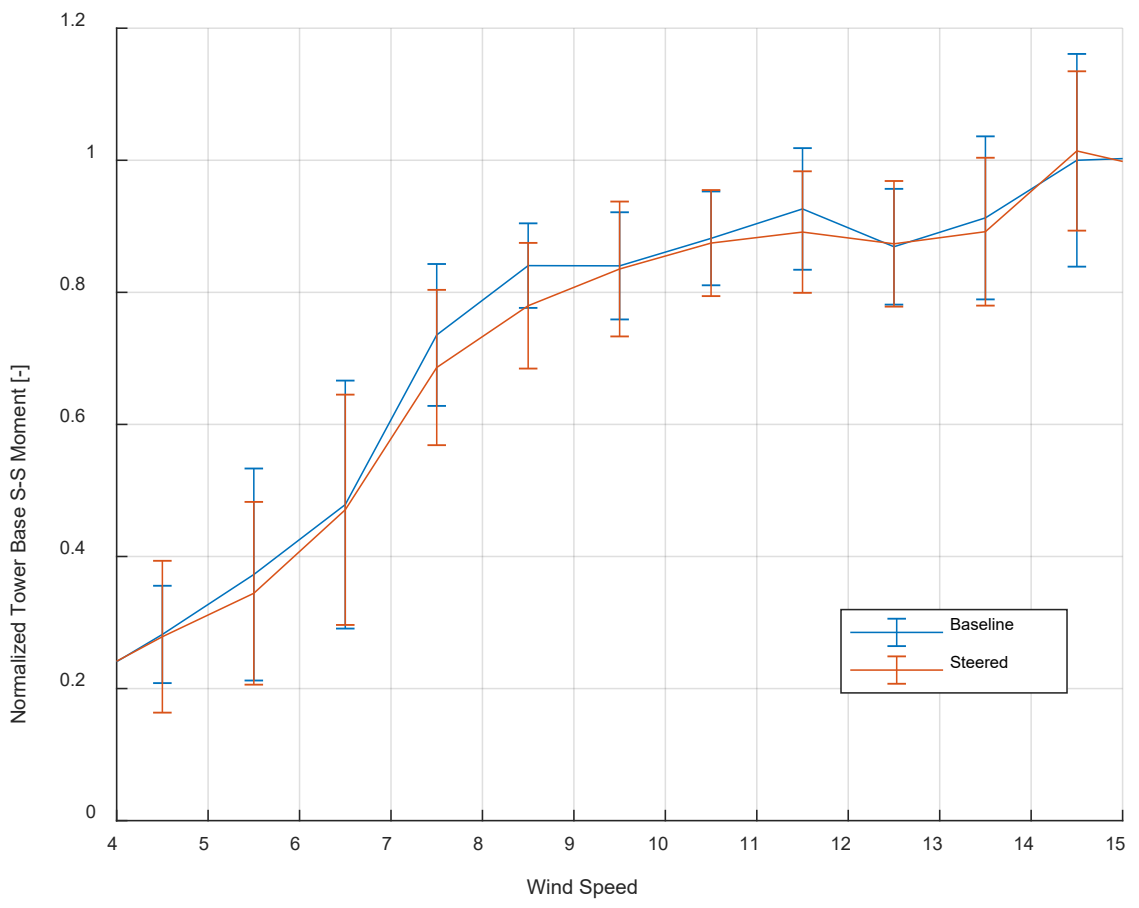

Figure 29. Comparison of normalized tower base side-side binned short-term (10-minute) damage equivalent loads

Error bars are $+/-1 \sigma$. 


\section{Conclusions}

This field campaign was intended to help understand the impact of wake steering controls applied to a wind farm. This report summarizes the results of a mechanical loads experiment that aimed to measure the impact of a wind turbine that is downwind from another turbine with applied wake steering controls.

Although both turbines were instrumented following guidance according to the International Electrotechnical Commission's (IEC's) standard Wind Turbine Generator Systems - Part 13: Measurement of Mechanical Loads (IEC 61400-13, Edition 1.0, 2015) [4], or the Standard, only the loads of the downwind non-steered turbine were shown here (T3). Data were collected by switching the wake steering controls on T2 on/off incrementally following a predetermined pattern. This allowed the baseline and steered databases to have statistically meaningful amounts of data while keeping the turbulence intensity levels very similar.

Following data collection and quality control, a loads analysis was conducted in accordance with the Standard, and figures were plotted to summarize the statistics. Overall, the binned statistics showed minimal differences in loading between the baseline and steered cases. Upon examining the fatigue analysis damage equivalent loads, the wake steering cases exhibit reduced fatigue for most components. Additionally, results showed significant scatter in Region 2 for both cases, likely because of turbulence, which can influence the outcomes of expected trends.

While this analysis did not specifically investigate the influence of partial versus full wake or other atmospheric influences it provides meaningful insights into the efficacy of wake steering controls at a plant level and implies that the downwind turbine experiences an overall reduction in fatigue loads - a benefit to the turbine operating life. 


\section{Future Work}

The rich database that was compiled during this field campaign holds value for further analyses and additional insights. Notable topics for future research include:

- A more granular yaw offset loads analysis to better understand load and fatigue sensitivity to wake steering controls.

- Incorporation of an upwind (T2) turbine loads analysis for a net loads study. Additionally, studying the loads sensitivity to different wind directions within the measurement sector could bring more insights to influences from partial versus full wakes.

- A more in-depth study of fatigue to help quantify the difference in loading, possibly by (1) analyzing the lifetime fatigue in the context of the turbine pair examined for this campaign and (2) creating a spectrum by varying the distribution of wake steering operation (e.g. incrementally increasing the amount of time wake steering controls are applied over the design life of the turbine compared to normal turbine controls and operations). 


\section{References}

[1] R. Damiani et al., "Assessment of wind turbine component loads under yaw-offset conditions," Wind Energy Sci., vol. 3, no. 1, pp. 173-189, Apr. 2018, doi: https://doi.org/10.5194/wes-3-173-2018.

[2] P. Fleming et al., "Initial results from a field campaign of wake steering applied at a commercial wind farm - Part 1," Wind Energy Sci., vol. 4, no. 2, pp. 273-285, May 2019, doi: https://doi.org/10.5194/wes-4-273-2019.

[3] P. Fleming et al., "Continued results from a field campaign of wake steering applied at a commercial wind farm - Part 2," Wind Energy Sci., vol. 5, no. 3, pp. 945-958, Jul. 2020, doi: https://doi.org/10.5194/wes-5-945-2020.

[4] "Wind turbines - Part 13: Measurement of mechanical loads," IEC 61400-13, Edition 1.0, 2015.

[5] G. Hayman and M. Buhl, Jr., Mlife. NREL, 2012.

[6] H. Mendez Reyes, S. Kanev, B. Doekemeijer, and J.-W. van Wingerden, "Validation of a lookup-table approach to modeling turbine fatigue loads in wind farms under active wake control," Wind Energy Sci., vol. 4, no. 4, pp. 549-561, Oct. 2019, doi:

https://doi.org/10.5194/wes-4-549-2019. 


\section{Appendix A. Coordinate Systems}

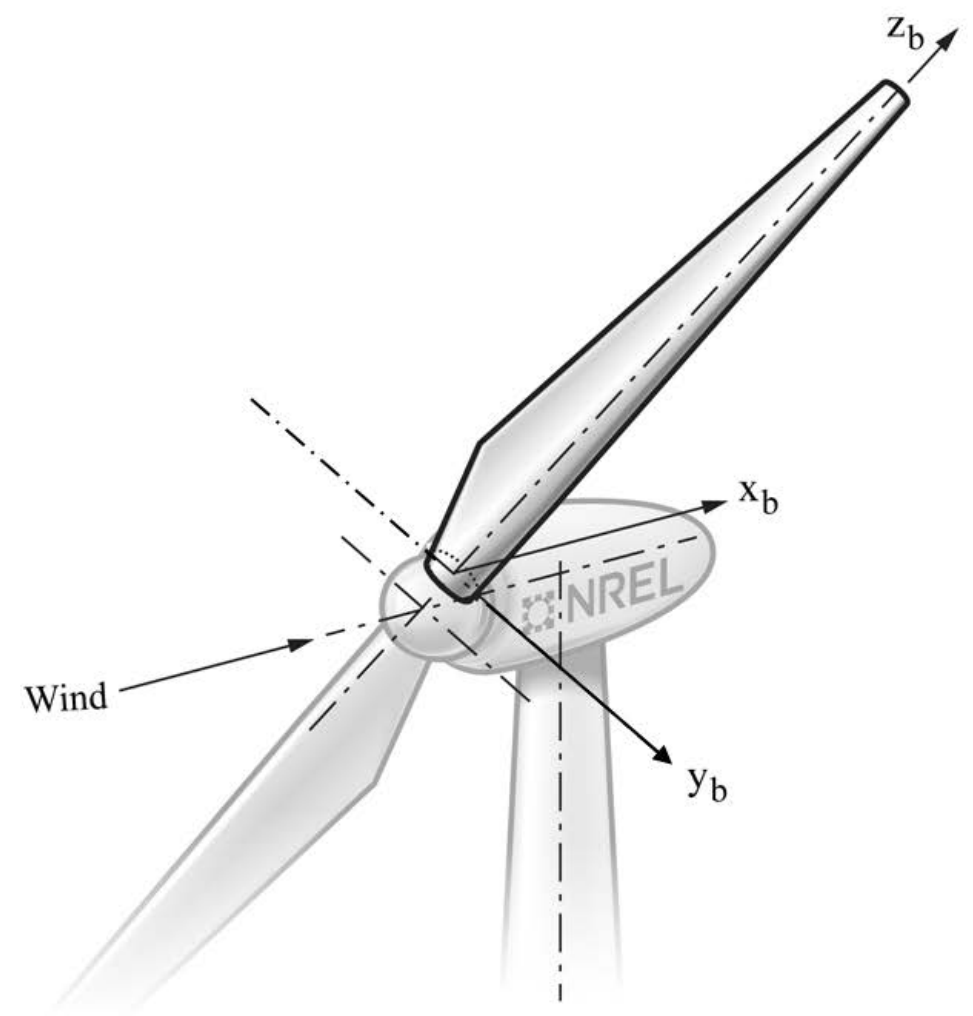

Figure 30. Blade root coordinate system

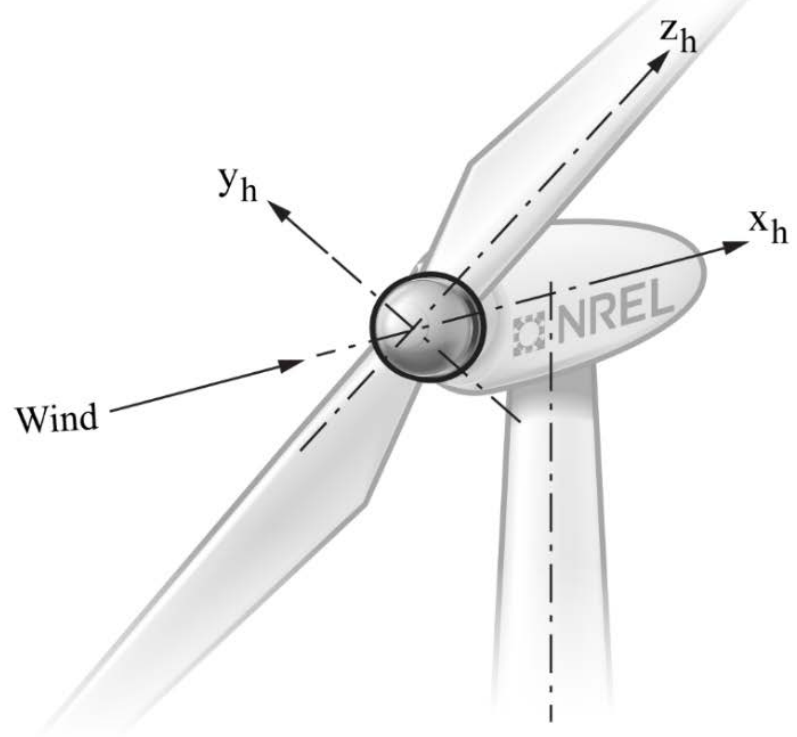

Figure 31. Rotor hub coordinate system 


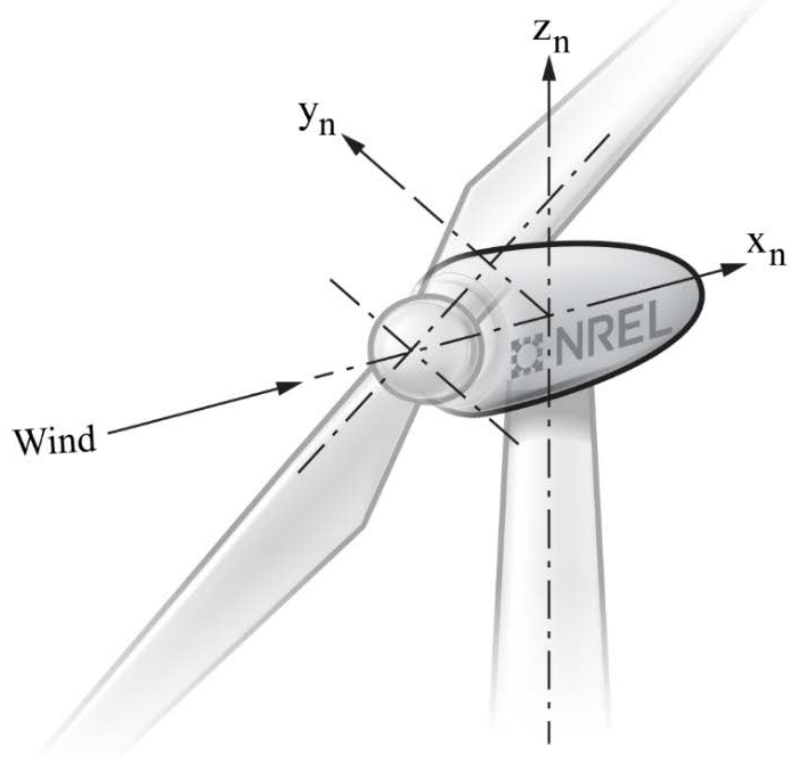

Figure 32. Nacelle coordinate system

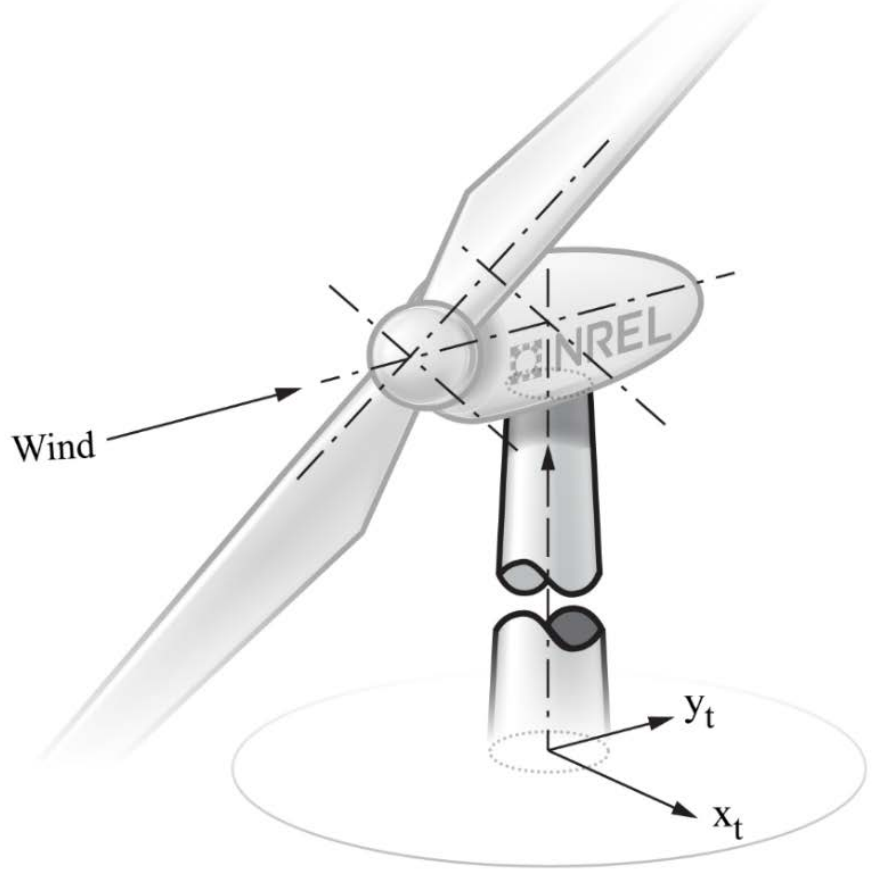

Figure 33. Tower coordinate system 


\section{Appendix B. Component Loads Statistical Scatter Plots}
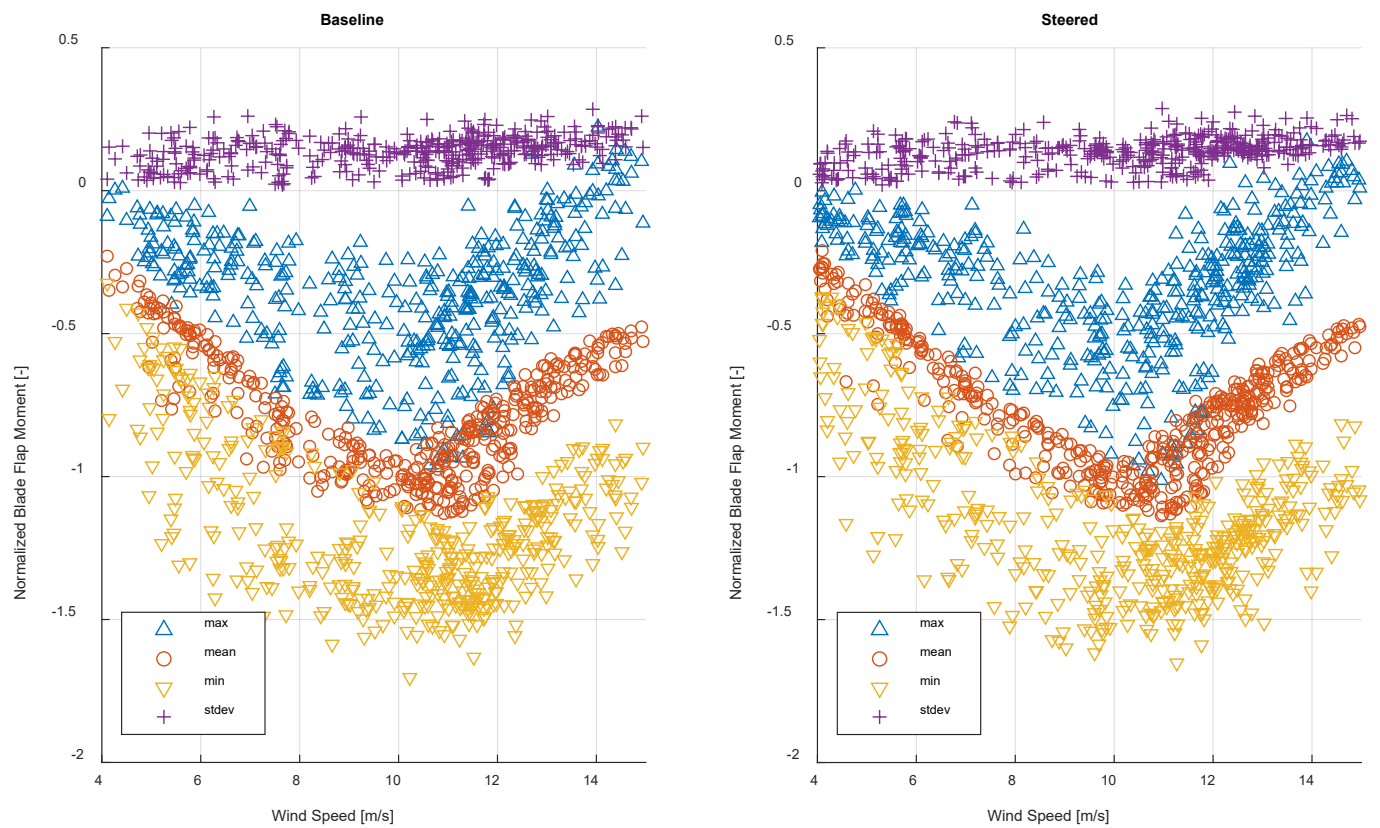

Figure 34. Comparison of 10-min statistics for baseline and steered blade 1 root flapwise bending moments normalized by absolute max of mean baseline binned statistic
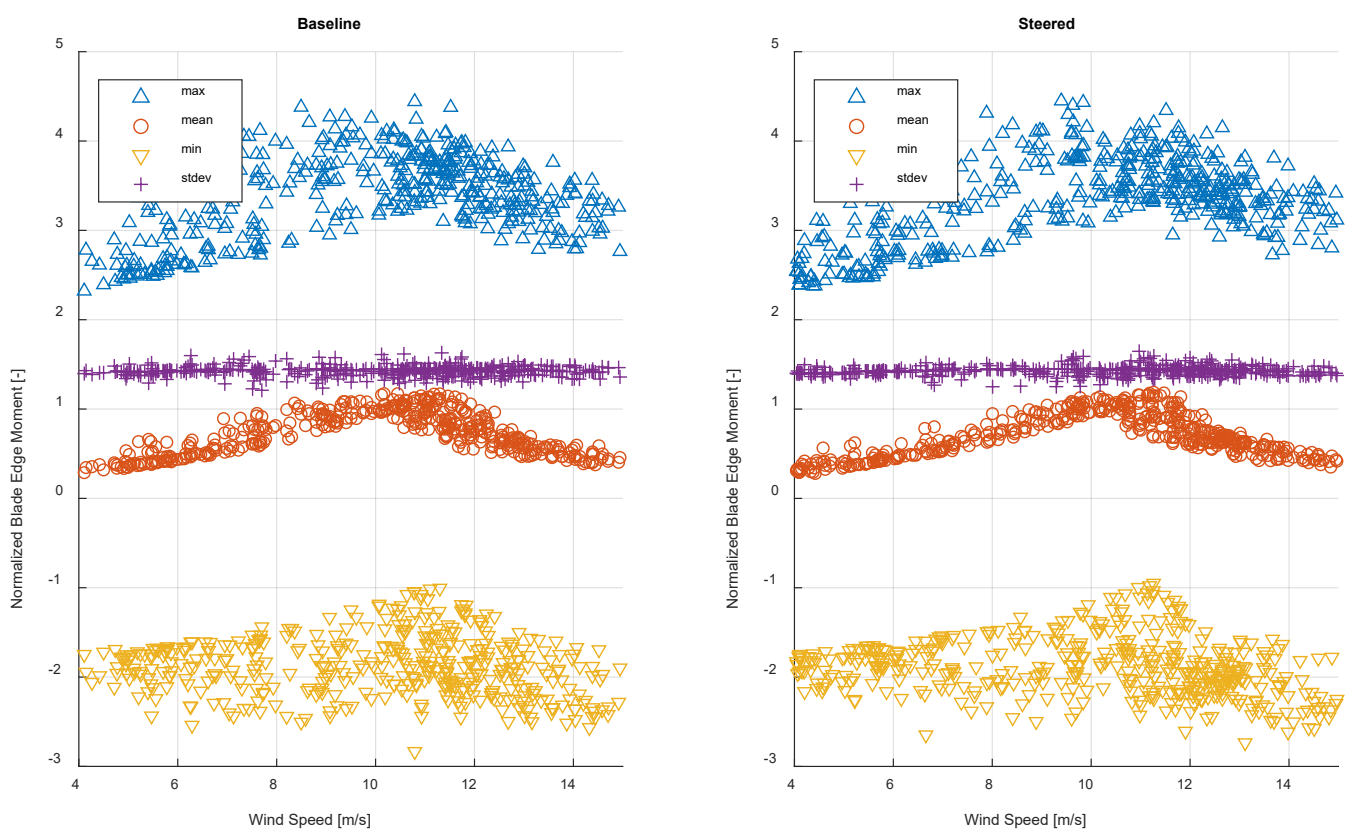

Figure 35. Comparison of 10-min statistics for baseline and steered blade 1 root edgewise bending moments normalized by absolute max of mean baseline binned statistic 

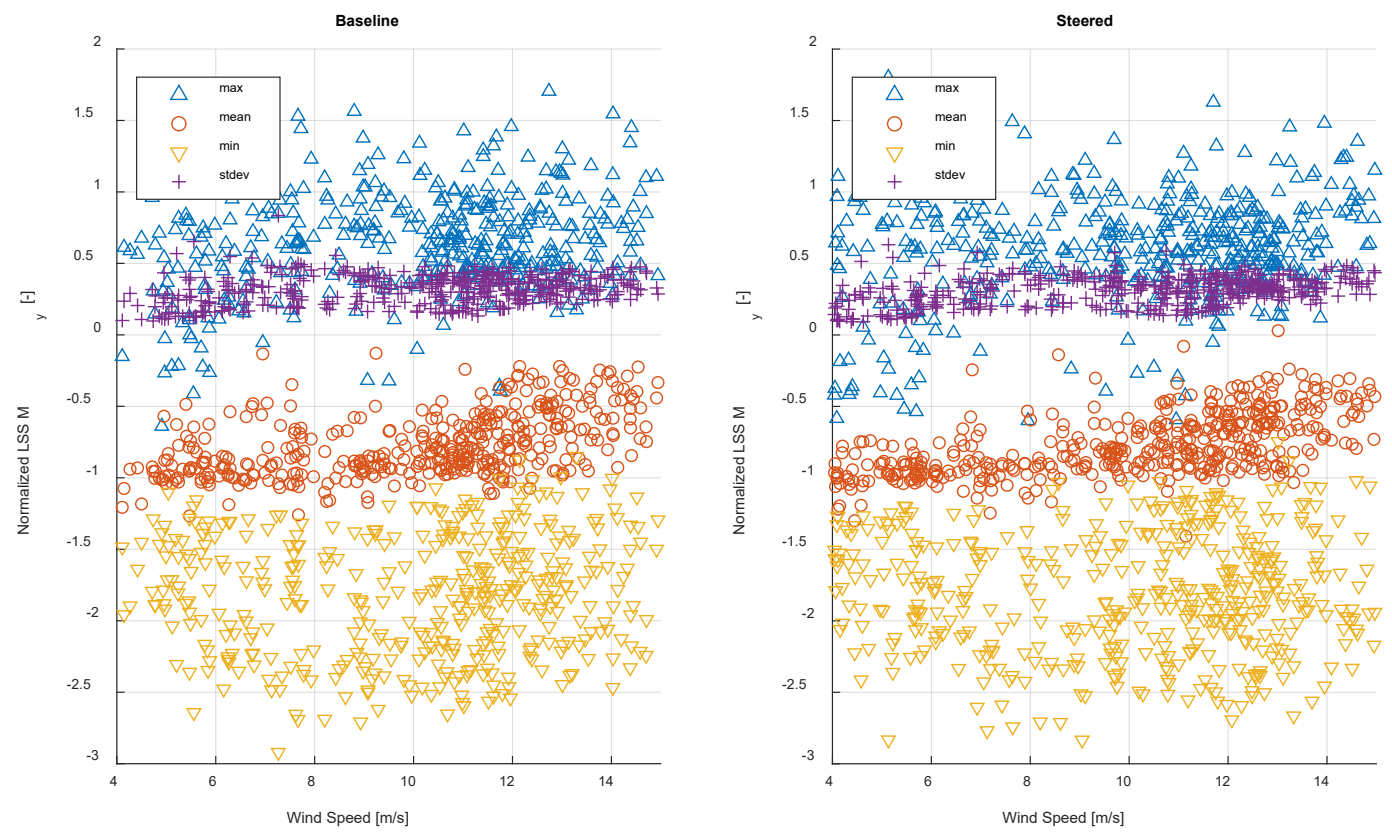

Figure 36. Comparison of 10-min statistics for baseline and steered main shaft $\left(M_{y}\right)$ bending moments normalized by absolute max of mean baseline binned statistic
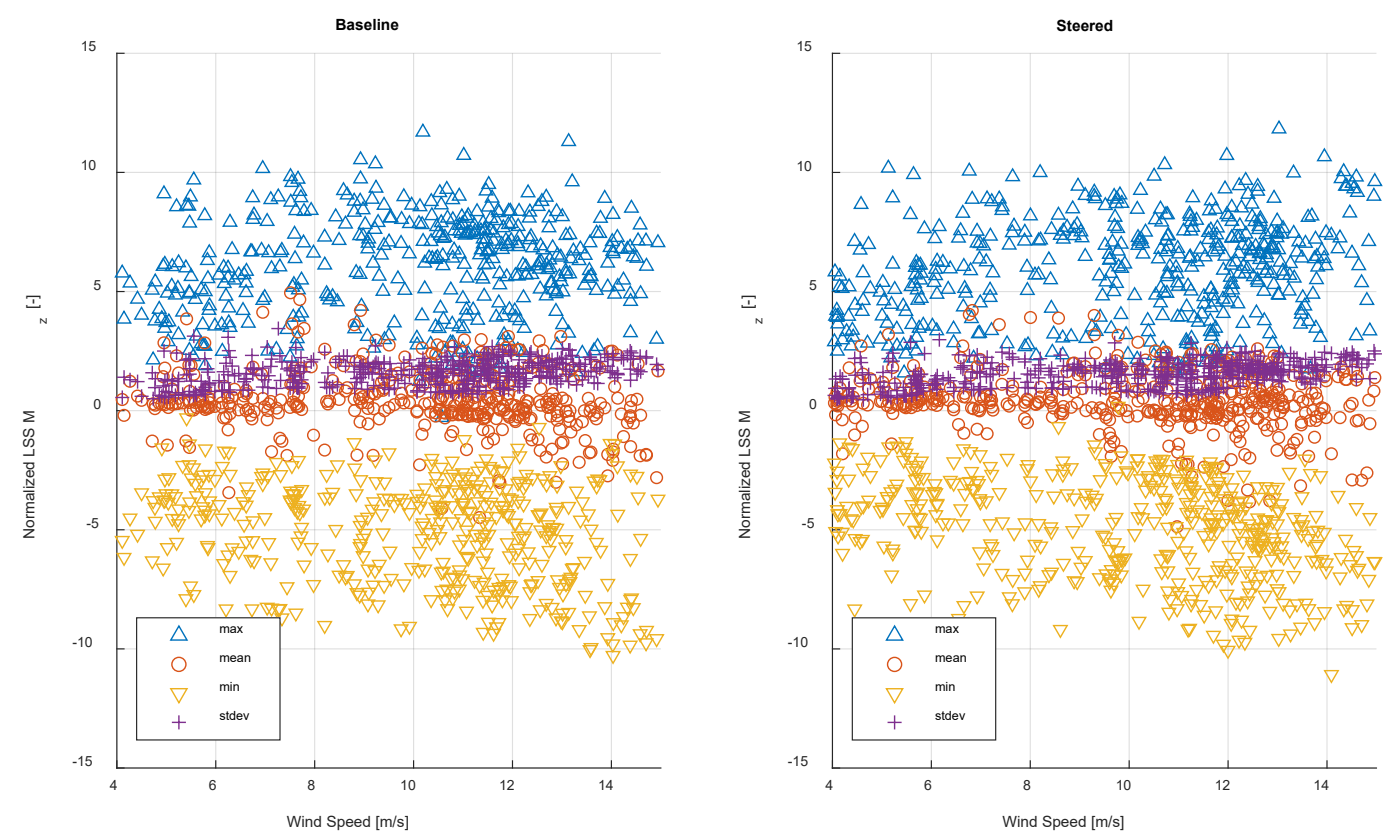

Figure 37. Comparison of 10-min statistics for baseline and steered main shaft $\left(M_{z}\right)$ bending moments normalized by absolute max of mean baseline binned statistic 

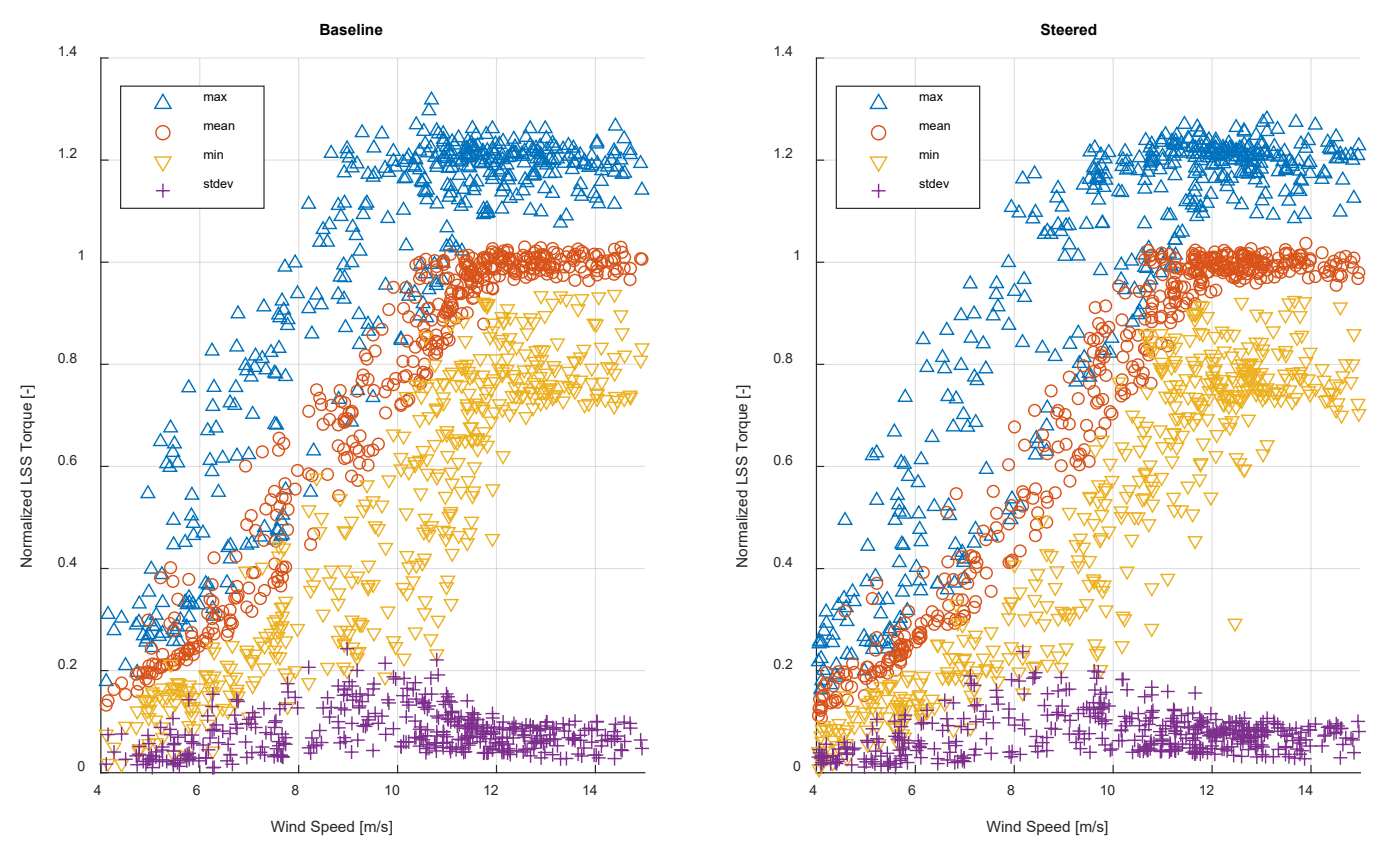

Figure 38. Comparison of 10-min statistics for baseline and steered main shaft torque response normalized by absolute max of mean baseline binned statistic
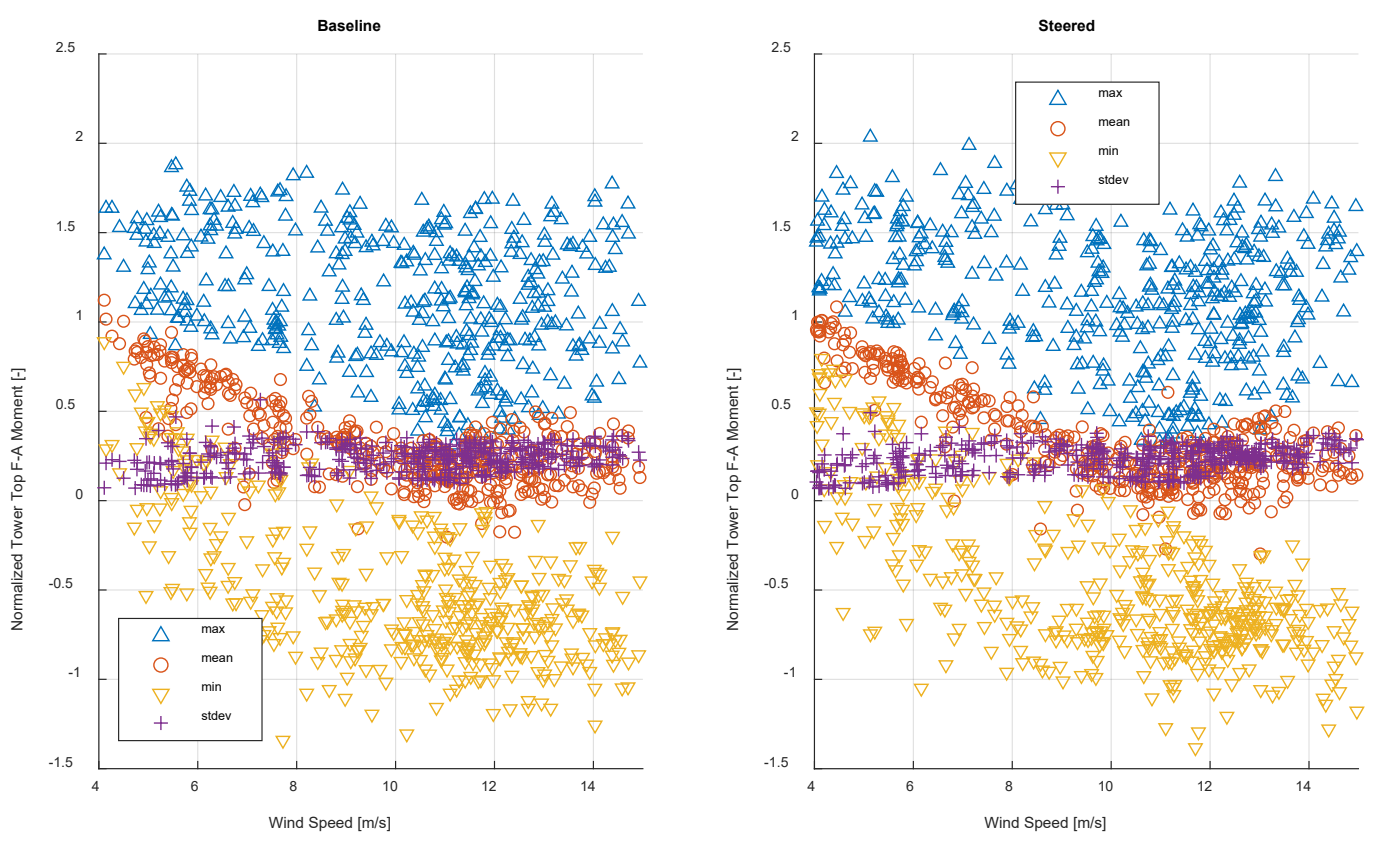

Figure 39. Comparison of 10-min statistics for baseline and steered tower top fore-aft bending moments normalized by absolute max of mean baseline binned statistic 

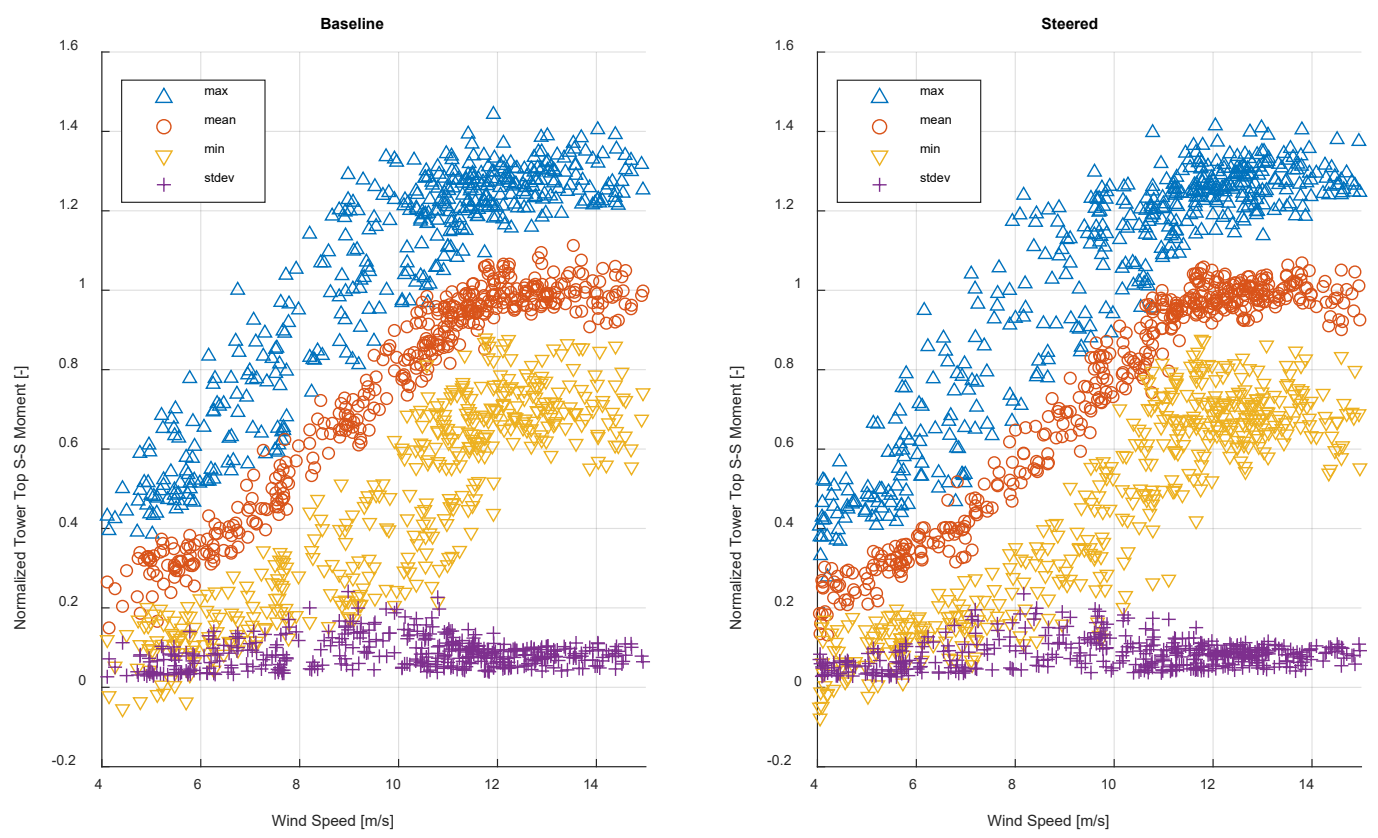

Figure 40. Comparison of 10-min statistics for baseline and steered tower top side-side bending moments normalized by absolute max of mean baseline binned statistic
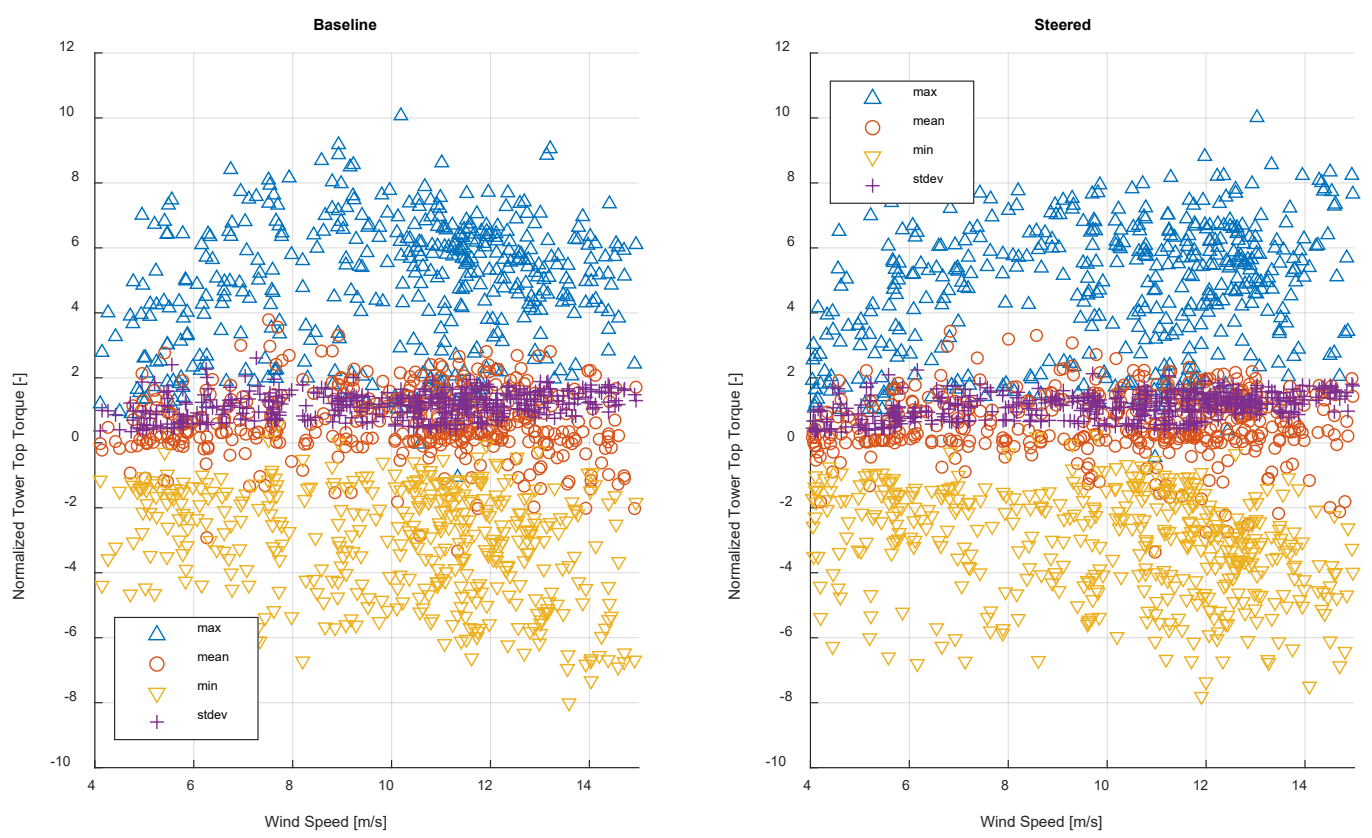

Figure 41. Comparison of 10-min statistics for baseline and steered tower top torque response normalized by absolute max of mean baseline binned statistic 

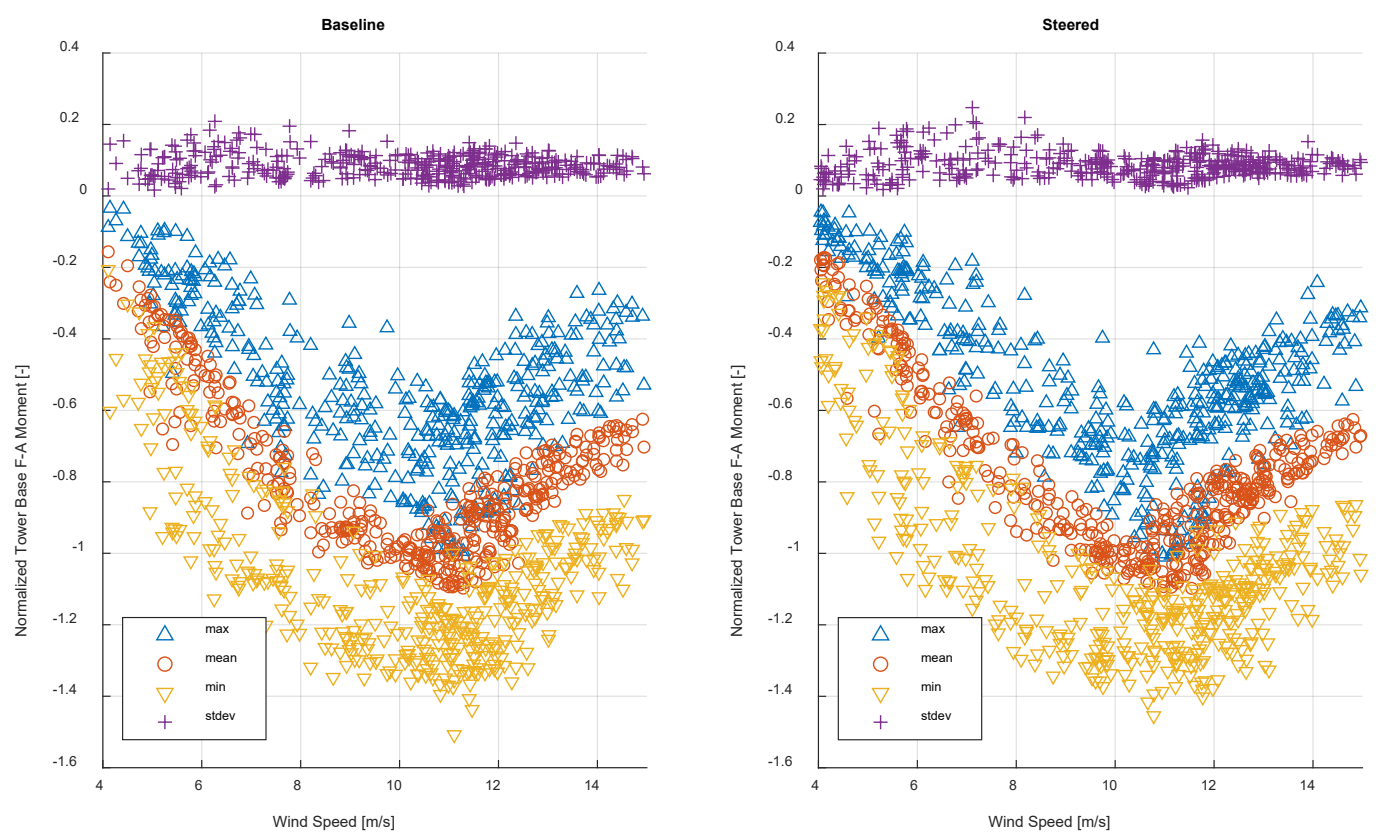

Figure 42. Comparison of 10-min statistics for baseline and steered tower base fore-aft bending moments normalized by absolute max of mean baseline binned statistic
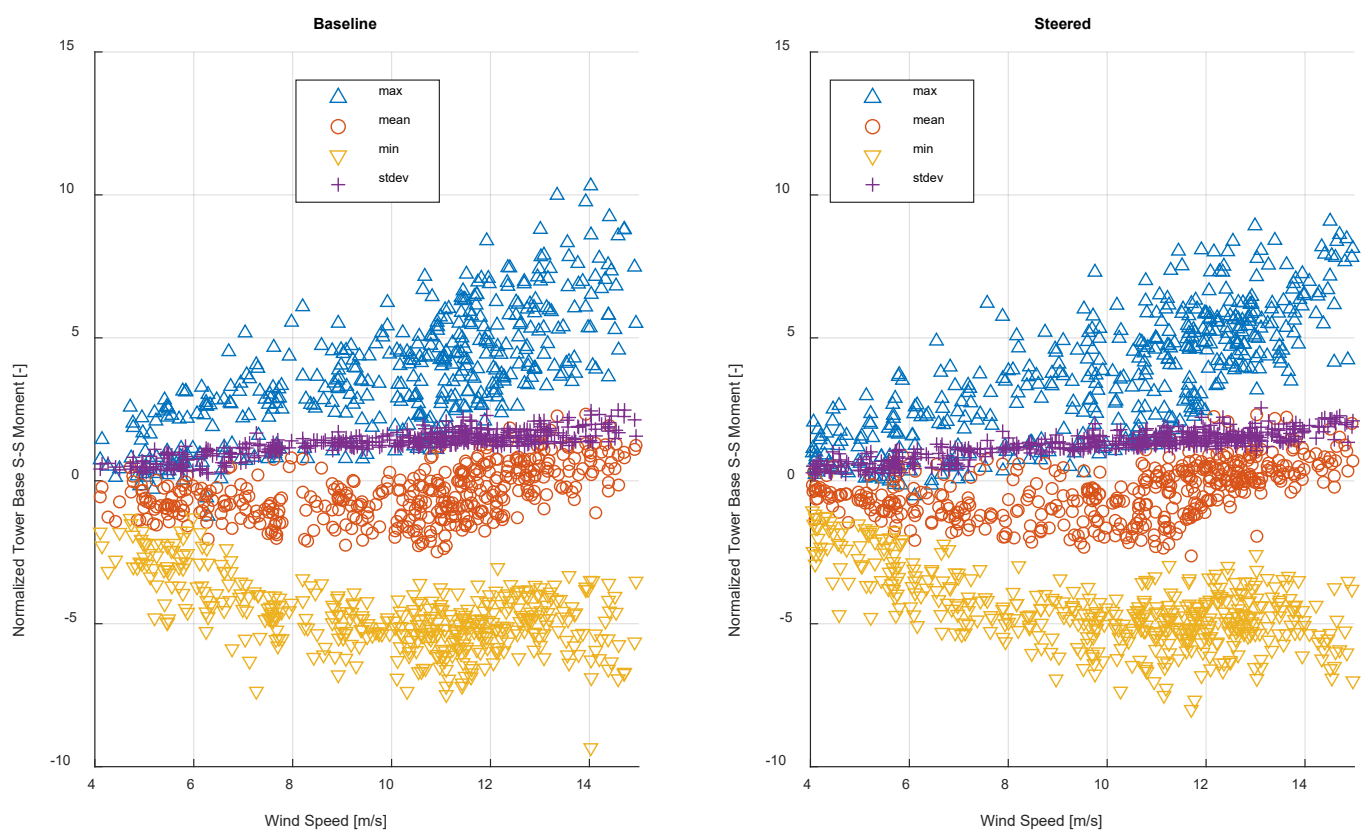

Figure 43. Comparison of 10-min statistics for baseline and steered tower base side-side bending moments normalized by absolute max of mean baseline binned statistic 


\section{Appendix C. Damage Equivalent Loads Scatter Plots}

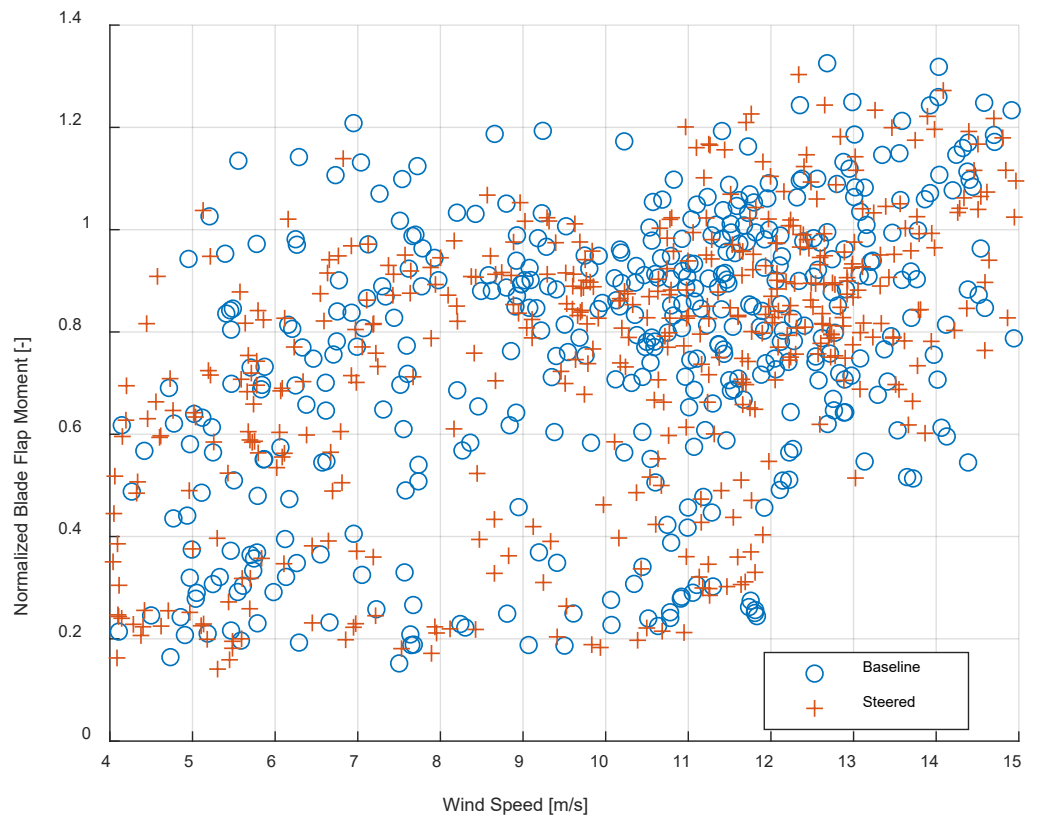

Figure 44. Damage equivalent load comparison of baseline and steered cases for blade 1 root flapwise bending moments normalized by the absolute max value of the baseline binned DEL

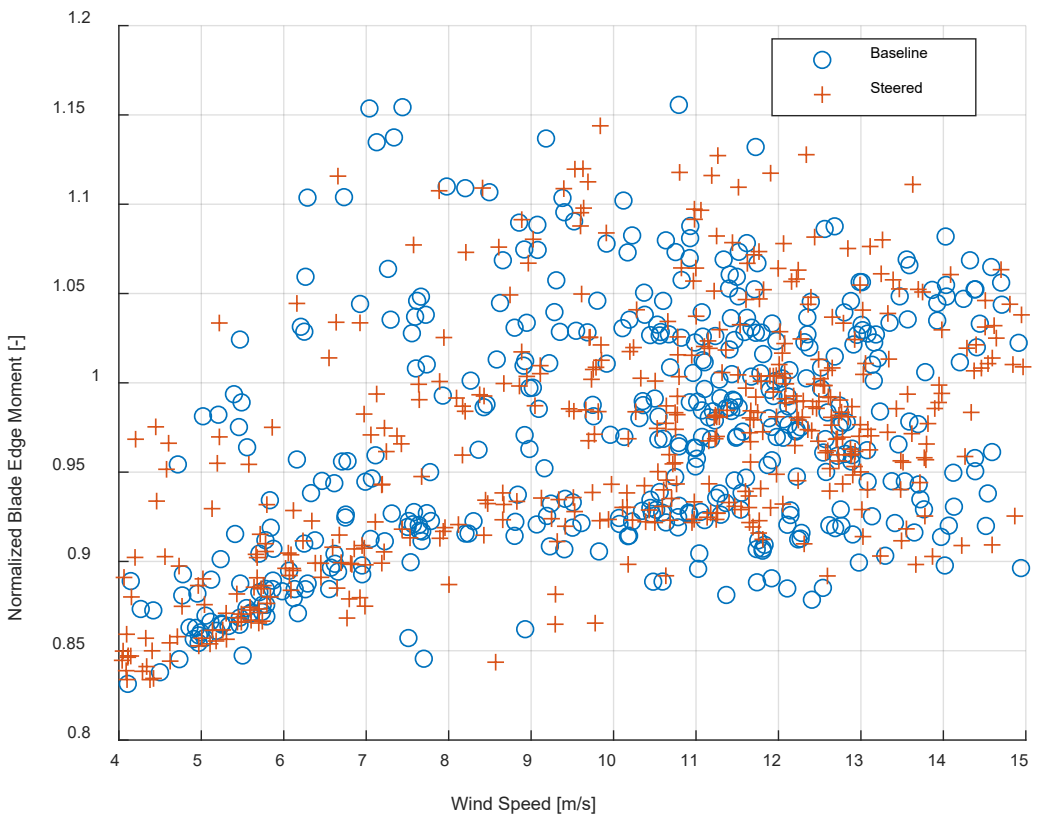

Figure 45. Damage equivalent load comparison of baseline and steered cases for blade 1 root edgewise bending moments normalized by the absolute max value of the baseline binned DEL 


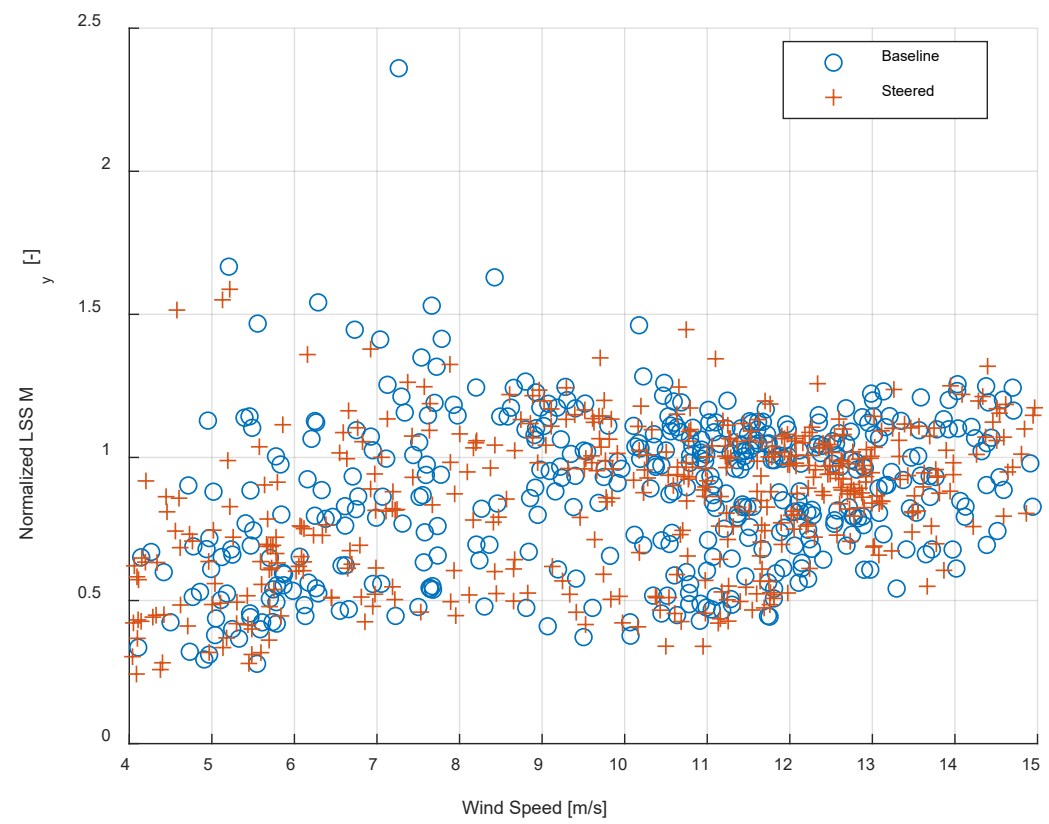

Figure 46. Damage equivalent load comparison of baseline and steered cases for main shaft ( $\left.M_{y}\right)$ bending moments normalized by the absolute max value of the baseline binned DEL

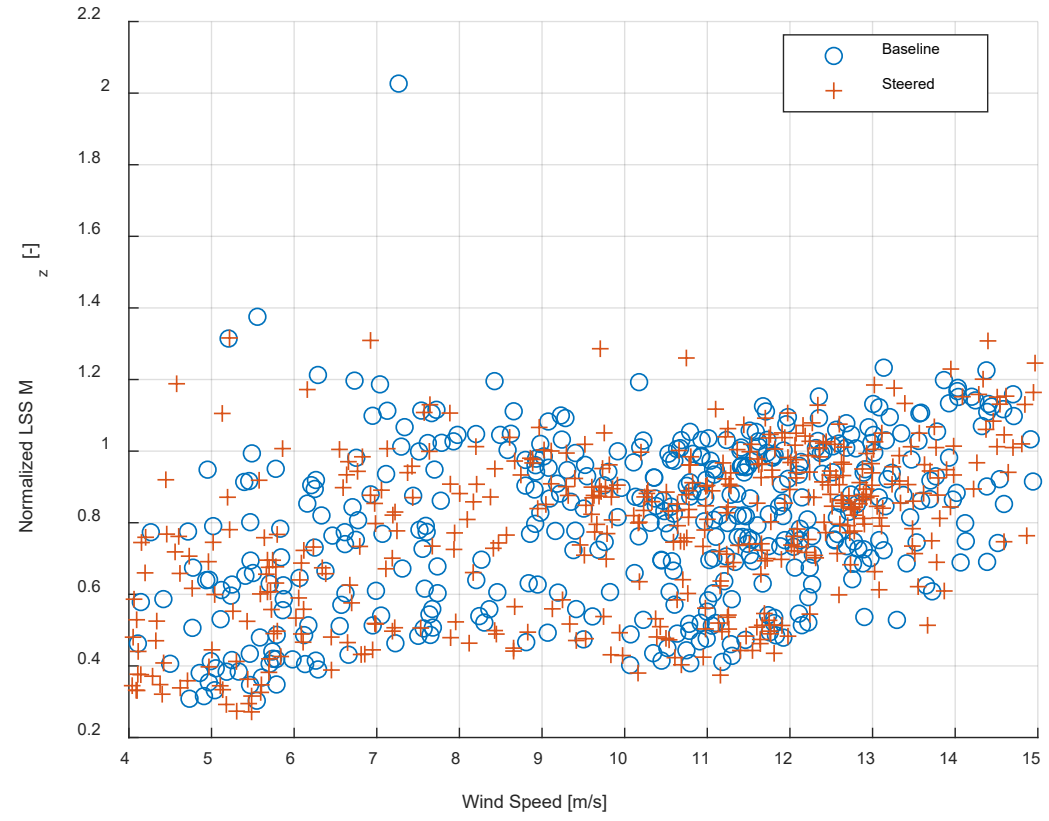

Figure 47. Damage equivalent load comparison of baseline and steered cases for main shaft $\left(M_{z}\right)$ bending moments normalized by the absolute max value of the baseline binned DEL 


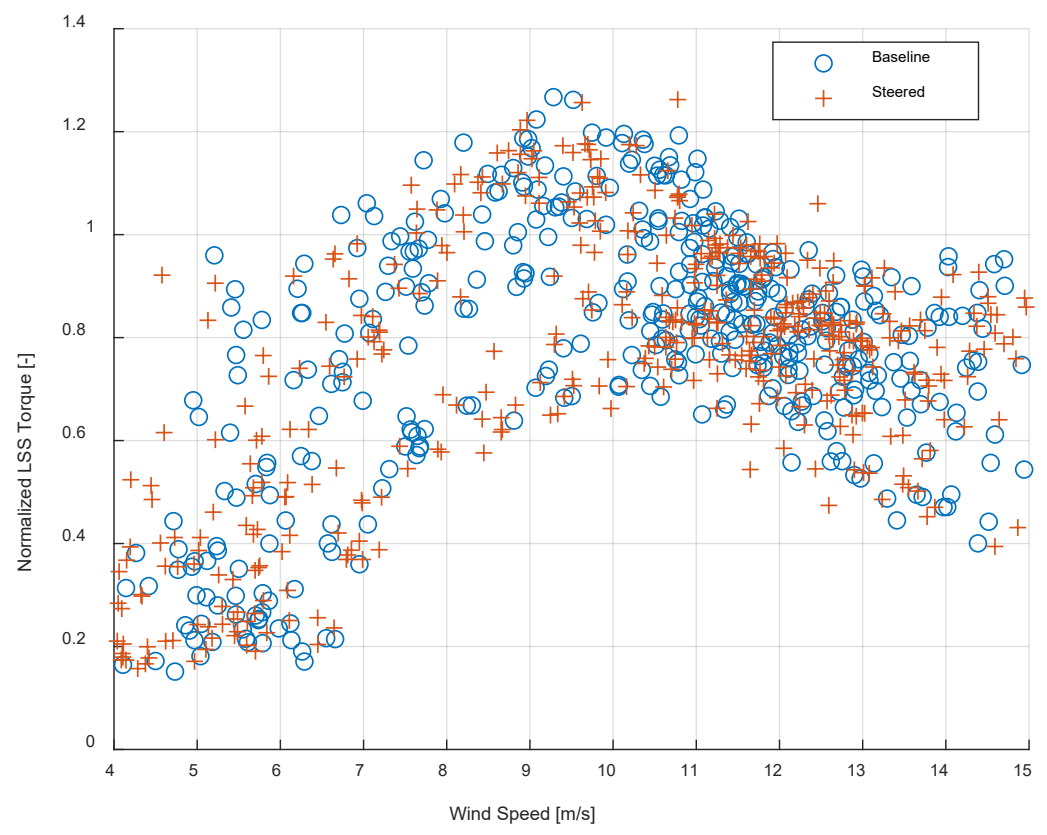

Figure 48. Damage equivalent load comparison of baseline and steered cases for main shaft torque response normalized by the absolute max value of the baseline binned DEL

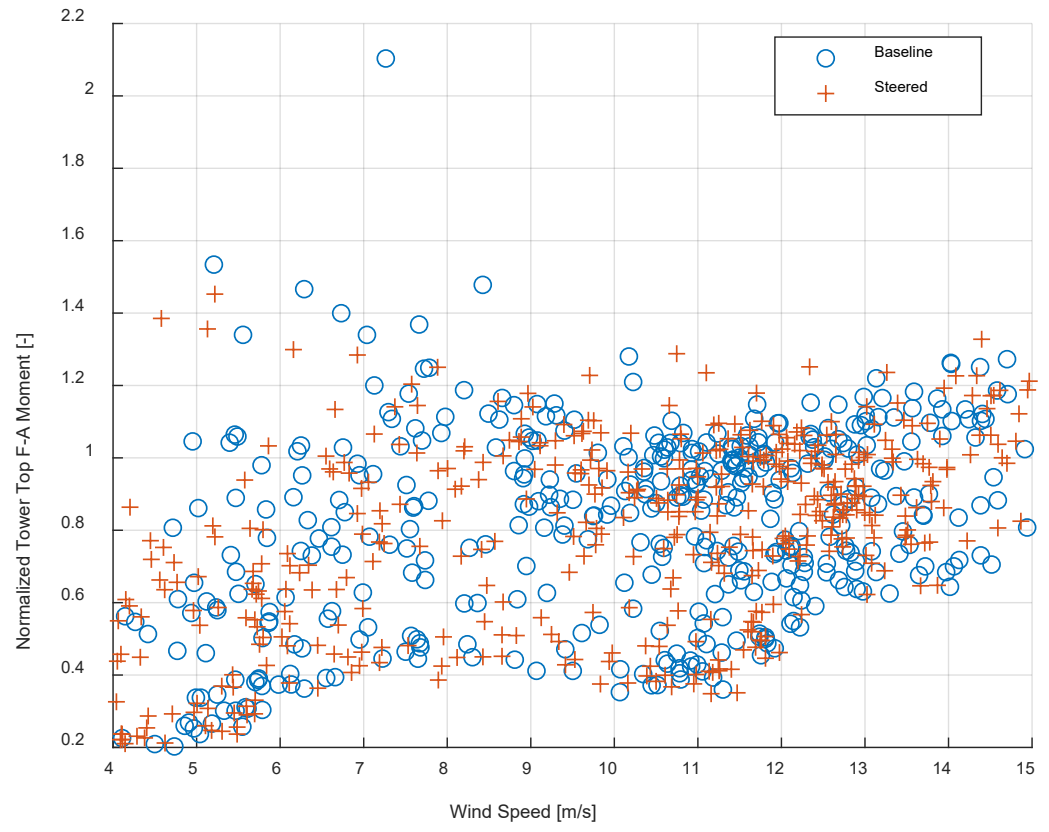

Figure 49. Damage equivalent load comparison of baseline and steered cases for tower top foreaft bending moments normalized by the absolute max value of the baseline binned DEL 


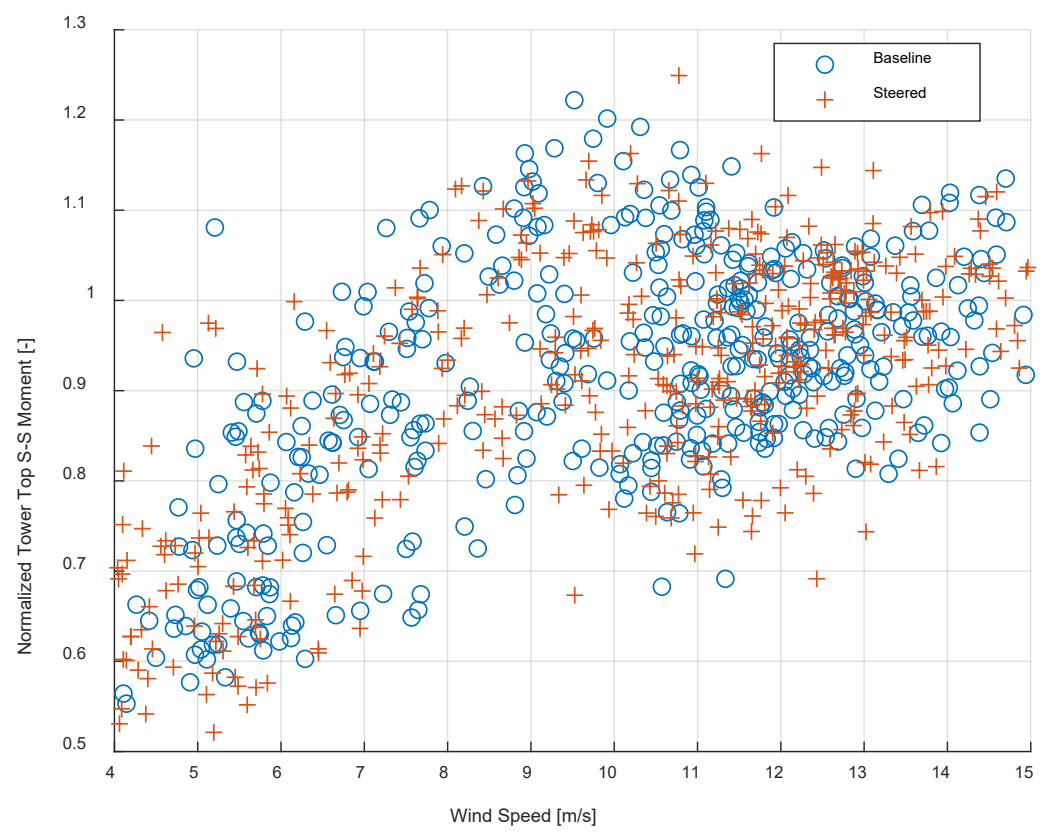

Figure 50. Damage equivalent load comparison of baseline and steered cases for tower top sideside bending moments normalized by the absolute max value of the baseline binned DEL

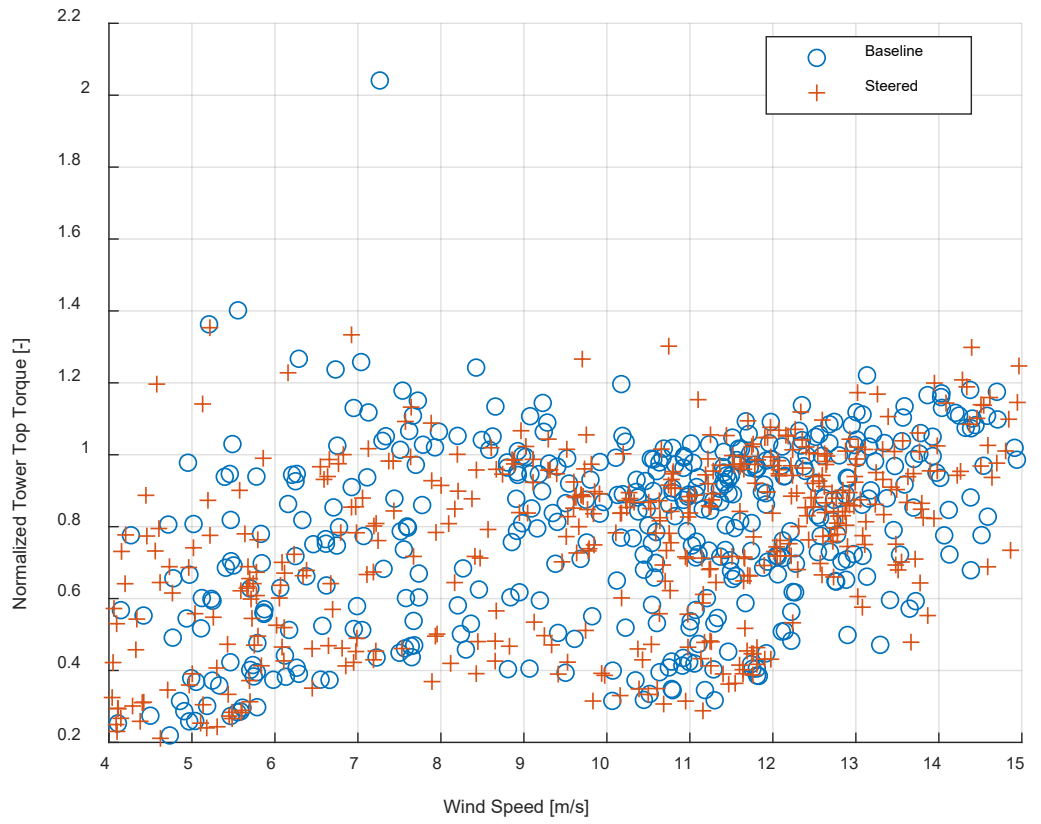

Figure 51. Damage equivalent load comparison of baseline and steered cases for tower top torque response normalized by the absolute max value of the baseline binned DEL 


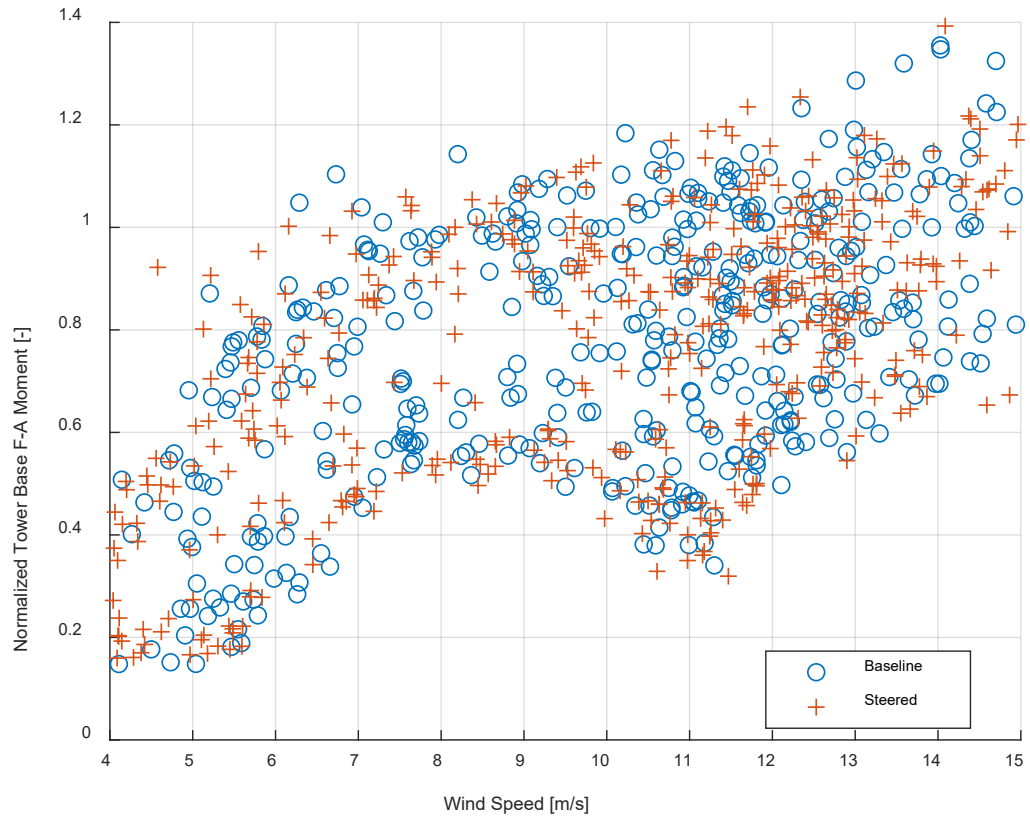

Figure 52. Damage equivalent load comparison of baseline and steered cases for tower base foreaft bending moments normalized by the absolute max value of the baseline binned DEL

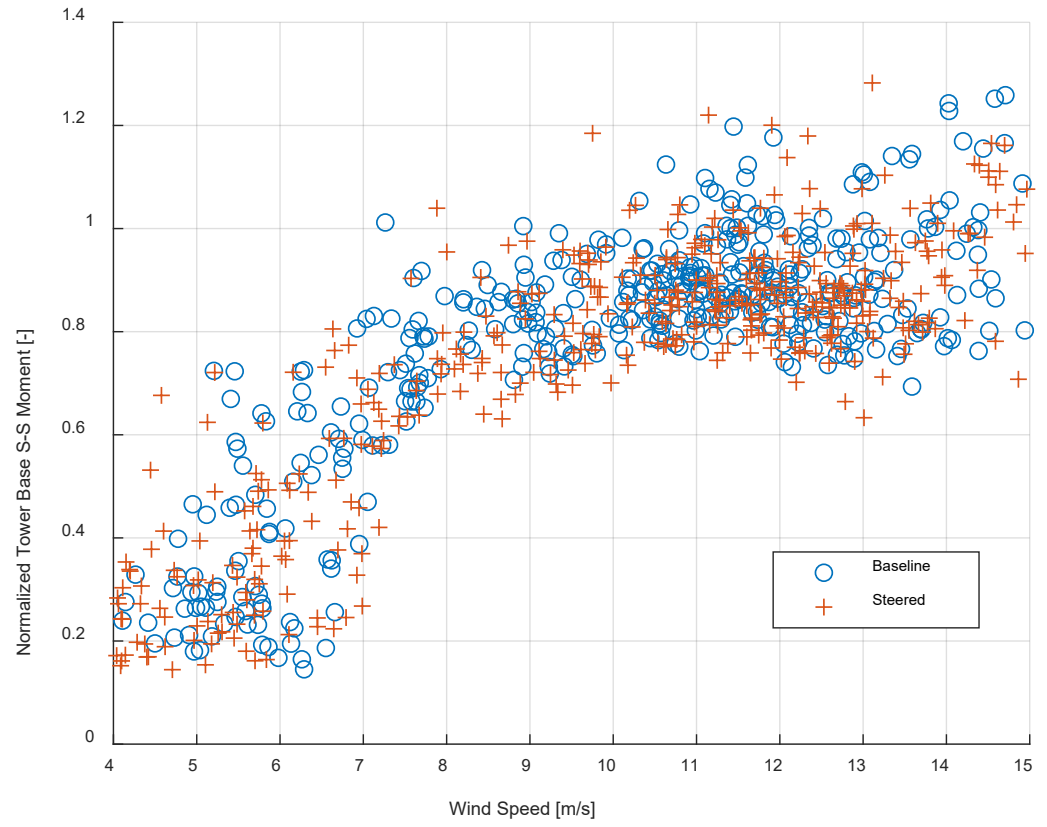

Figure 53. Damage equivalent load comparison of baseline and steered cases for tower base sideside bending moments normalized by the absolute max value of the baseline binned DEL 